\title{
Feasibility of spectro-polarimetric characterization of exoplanetary atmospheres with direct observing instruments
}

\author{
J. Takahashi ${ }^{1}$, T. Matsuo ${ }^{2}$, and Y. Itoh ${ }^{1}$ \\ 1 Nishi-Harima Astronomical Observatory, Center for Astronomy, University of Hyogo, 407-2, Nishigaichi, Sayo, 679-5313 Hyogo, \\ Japan \\ e-mail: takahashi@nhao.jp \\ 2 Department of Earth and Space Science, Graduate School of Science, Osaka University 1-1, Machikaneyama-Cho, Toyonaka, \\ 560-0043 Osaka, Japan
}

Received 28 January 2016 / Accepted 9 November 2016

\begin{abstract}
Context. Spectro-polarimetry of reflected light from exoplanets is anticipated to be a powerful method for probing atmospheric composition and structure.

Aims. We aim to evaluate the feasibility of the search for a spectro-polarimetric feature of water vapor using a high-contrast polarimetric instrument on a 30-40 m-class ground-based telescope.

Methods. Three types of errors are considered: (a) errors from the difference between efficiencies for two orthogonally polarized states; (b) errors caused by speckle noises; and (c) errors caused by photon noise from scattered starlight. Using the analytically derived error formulas, we estimate the number of planets for which feasible spectro-polarimetric detection of water vapor is possible. Results. Our calculations show that effective spectro-polarimetric searches for water vapor are possible for 5 to 14 known planets. Spectro-polarimetric characterization of exoplanetary atmospheres is feasible with an extremely large telescope and a direct observing spectro-polarimeter.
\end{abstract}

Key words. planetary systems - techniques: polarimetric - instrumentation: polarimeters

\section{Introduction}

Spectro-polarimetric observations of visible or near-infrared reflected light from exoplanets can be a powerful tool for probing their atmospheres. At molecular absorption wavelengths, the polarization degree of planetary reflected light increases owing to the decrease in intensity of the multiply scattered component as compared with that of the continuum wavelengths. Stam et al. (2004) and Stam (2008) calculated polarization degree spectra for Jupiter-like and Earth-like planets, respectively, and derived enhanced features due to absorption by atmospheric molecules such as $\mathrm{CH}_{4}, \mathrm{H}_{2} \mathrm{O}$, and $\mathrm{O}_{2}$. In fact, Sterzik et al. (2012) and Miles-Páez et al. (2014) clearly detected $\mathrm{H}_{2} \mathrm{O}$ (at wavelengths $0.72,0.82,0.93$, and $1.12 \mu \mathrm{m})$ and $\mathrm{O}_{2}(0.69,0.76$, and $1.25 \mu \mathrm{m})$ features on the polarization spectra of lunar Earthshine. Hence, spectro-polarimetry for exoplanets can be used for the detection of these atmospheric molecules.

Spectro-polarimetry facilitates not only the detection of atmospheric molecules, but also further characterization of the atmosphere. Stam et al. (2004) and Stam (2008) showed that the polarization of planets is sensitive to the structure of the atmosphere. Stam et al. (2004) simulated three different model atmospheres of Jupiter-like planets: (1) an atmosphere that contains only gaseous molecules ("clear atmosphere"); (2) an atmosphere with a tropospheric cloud layer ("low cloud atmosphere"); and (3) an atmosphere with a tropospheric cloud layer and a stratospheric haze layer ("high haze atmosphere"). The resulting differences between the polarization spectra from the models were significant in overall wavelength-dependence and strength of the molecular features, whereas the differences in the intensity spectra were less clear. Similarly, Stam (2008) pointed out that the degree of polarization at the continuum wavelengths near a molecular feature is more sensitive to the altitude of the cloud's top compared with intensity. Therefore, spectro-polarimetry provides information that cannot be obtained solely by intensity spectroscopy.

In addition to these scientific benefits, polarimetry has some technical advantages. Because polarimetry is a relative measurement, the degree (and position angle) of polarization is not affected by isotropic transmittance between celestial objects and observers. This advantage will be especially effective for ground-based detection of $\mathrm{H}_{2} \mathrm{O}, \mathrm{O}_{2}$, and other components common with the Earth's atmosphere, if the telluric transmittance is isotropic.

The degree of polarization $P$ of a planet is derived by the equation:

$P(\lambda)=\frac{I_{0}^{\mathrm{p}}(\lambda)-I_{90}^{\mathrm{p}}(\lambda)}{I_{0}^{\mathrm{p}}(\lambda)+I_{90}^{\mathrm{p}}(\lambda)}$,

where $I_{0}^{\mathrm{p}}$ and $I_{90}^{\mathrm{p}}$ represent planetary intensities of light oscillating perpendicular and parallel to the scattering plane (the plane which includes the central star, the planet, and the observer), respectively, and $\lambda$ is the wavelength. If the instrumental reference plane is aligned to the scattering plane (using a half-wave plate), we have Stokes parameters $U=0$ and $Q=P$, because scattering or reflected light is polarized perpendicular or parallel to the scattering plane. We take $I_{0}^{\mathrm{p}}$ and $I_{90}^{\mathrm{p}}$ as the values that are not altered by any absorption. When observations are conducted at the ground, intensities are affected by telluric transmittance. 
(a)

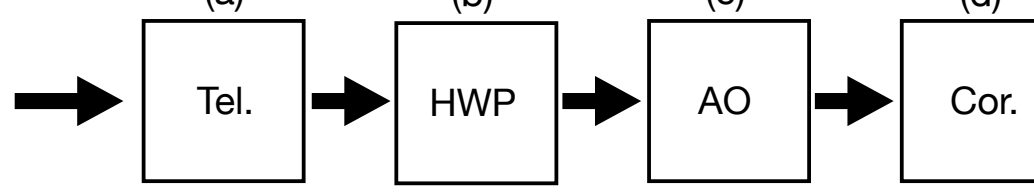

(e)

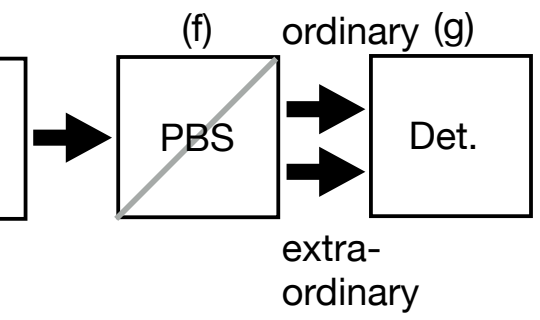

Fig. 1. Schematic illustration of the hypothetical observational system considered in this paper. It consists of a) a telescope; b) a half-wave plate; c) extreme adaptive optics; d) a coronagraph or a nulling interferometer; e) an integral field spectrograph; f) a polarizing beam splitter; and g) a detector.

Previously, it was believed that telluric transmittance was isotropic, and thus did not affect the polarization state of light from an astronomical object. However, Bailey et al. (2008) found that dust in the telluric atmosphere can produce polarization up to $\sim 5 \times 10^{-5}$. In addition, the polarization state is affected by interstellar transmittance, instrumental transmittance, and any offset error in the intensities. Therefore, we should express measured intensity values $\left(I^{\prime \mathrm{p}}\right)$ as

$$
\left(\begin{array}{r}
I^{\prime}{ }_{0}^{\mathrm{p}}(\lambda) \\
I_{90}^{\prime} \mathrm{p}(\lambda)
\end{array}\right)=\left(\begin{array}{r}
r_{0}(\lambda) \cdot I_{0}^{\mathrm{p}}(\lambda) \\
r_{90}(\lambda) \cdot I_{90}^{\mathrm{p}}(\lambda)
\end{array}\right)+\left(\begin{array}{r}
\epsilon_{0}(\lambda) \\
\epsilon_{90}(\lambda)
\end{array}\right),
$$

$r_{\chi}(\lambda)=\mathcal{S}_{\chi}(\lambda) \cdot \mathcal{T}_{\chi}(\lambda) \cdot k_{\chi}(\lambda) \quad\left(\chi=0^{\circ}, 90^{\circ}\right)$,

where $r_{\chi}$ is the total efficiency from the object to the detector for ordinary $\left(\chi=0^{\circ}\right)$ or extraordinary $\left(\chi=90^{\circ}\right)$ components. It is the product of interstellar transmittance $\left(\mathcal{S}_{\chi}\right)$, telluric transmittance $\left(\mathcal{T}_{\chi}\right)$, and instrumental throughput including detector efficiency $\left(k_{\chi}\right), \epsilon_{\chi}$ is any offset error in the measurement of intensity values. In this paper, we examine how $r_{\chi}$ and $\epsilon_{\chi}$ affect the measured degree of polarization.

In the 2020s, 30-40 m-class ground-based telescopes such as the Thirty-Meter Telescope (TMT), the European Extremely Large Telescope (E-ELT), and the Giant Magellan Telescope (GMT) will begin operation. Direct spectro-polarimetry or narrow-band imaging polarimetry for exoplanets is anticipated to be realized by a combination of high-contrast and polarimetric instruments on these extremely large telescopes (ELTs). Polarimetric differential imaging (PDI) is one of the highly effective techniques used to enhance the contrast range of an instrument and facilitate the detection of reflected light from planets around stars. PDI allows not only the detection of planets but also the measurement of polarization. PDI is widely utilized for currently operating instruments, such as HiCIAO (e.g., Tamura et al. 2006; Hashimoto et al. 2011; Tanii et al. 2012) on the Subaru telescope, and the SPHERE-ZIMPOL (e.g., Thalmann et al. 2008, 2015) and NACO (e.g., Lenzen et al. 2003; Rousset et al. 2003; Garufi et al. 2013) on the Very Large Telescopes (VLTs). A high-contrast direct-imaging polarimeter for exoplanets, EPICS-EPOL, is designed based on the ZIMPOL and is proposed for the E-ELT (Kasper et al. 2010; Keller et al. 2010). Furthermore, a concept of the spectro-polarimetric option for EPICS-EPOL has also been studied (Rodenhuis et al. 2012). A spectro-polarimeter based on their concept will enable an effective suppression of the residual speckles by combining PDI with simultaneous spectral differential imaging (SSDI) or chromatic speckle suppression (Marois et al. 2000; Sparks \& Ford 2002). Therefore, at this point, it is important to assess the feasibility of polarimetry using a high-contrast spectro-polarimeter with SSDI and PDI techniques.

In this paper, we evaluate the feasibility of spectropolarimetric characterization of exoplanetary atmospheres by taking into account the errors expected in high-contrast instruments. As shown Fig. 1, we consider a hypothetical spectropolarimeter based on the concept proposed by Rodenhuis et al. (2012). The instrument shall be equipped with an integral field spectrograph (IFS) and a polarizing beam splitter (PBS) to obtain a polarization degree spectrum of a planet. Although the EPISEPOL is a visible light instrument, we assume that our instrument works in the near-infrared because we focus on the detection of the strongest spectro-polarimetric feature of $\mathrm{H}_{2} \mathrm{O}$ at 1.12 $\mu \mathrm{m}$ on a visible-to-near-infrared spectrum (Miles-Páez et al. 2014). We assume that our instrument has a medium wavelength resolution $R$ of a few thousand. Although our target spectral feature is relatively broad $(\Delta \lambda \cong 0.05 \mu \mathrm{m}$, Miles-Páez et al. 2014), we follow the setting adopted by Barman et al. (2011) and Konopacky et al. (2013), who detected $\mathrm{CH}_{4}$ and $\mathrm{H}_{2} \mathrm{O}$ in exoplanet atmospheres by using the SSDI method for $R \sim$ 4000 data.

We focus on $\mathrm{H}_{2} \mathrm{O}$ vapor, which is a fundamental component in planetary atmospheres. $\mathrm{H}_{2} \mathrm{O}$ plays a major role in meteorology and habitability of Earth and other Solar System planets. Detection of $\mathrm{H}_{2} \mathrm{O}$ vapor has already been reported for several hot-Jupiters based on transit spectroscopy (e.g., Barman 2007; Tinetti et al. 2007; McCullough et al. 2014). However, transit spectroscopy is only possible for close-in planets. Direct observations can investigate planets in different orbital areas such as those near the habitable zone and the snow line. In addition, successful spectro-polarimetric searches for $\mathrm{a}_{2} \mathrm{O}$ feature will provide information on the structure of planetary atmospheres, such as cloud/haze height. Note that the framework we construct for $\mathrm{H}_{2} \mathrm{O}$ detectability evaluation can easily be applied to other atmospheric species.

We discuss the detectability of the $\mathrm{H}_{2} \mathrm{O}$ spectro-polarimetric feature with $\Delta P \equiv P\left(\lambda_{\mathrm{abs}}\right)-P\left(\lambda_{\mathrm{cnt}}\right)=10 \%$ and $P\left(\lambda_{\mathrm{cnt}}\right)=10 \%$, where $\lambda_{\mathrm{abs}}$ and $\lambda_{\mathrm{cnt}}$ represent absorption and its nearby continuum wavelengths, respectively $\left(\lambda_{\mathrm{abs}}=1.12 \mu \mathrm{m}\right)$. These values are based on lunar Earthshine observations by Miles-Páez et al. (2014) with an approximate correction of depolarization at the lunar surface (Earth pol. $\cong$ Earthshine pol. × 3, Dollfus 1957). Although our target exoplanets include gas-giants, the setting above is justified for the following reasons; (1) $\mathrm{H}_{2} \mathrm{O}$ vapor can be abundant if a planet is located within the snow line; and (2) as long as scattering of the atmosphere has a significant contribution to the total planetary intensity (i.e., clouds or hazes are not too high in altitude), a polarization degree spectrum of the planet is expected to have enhancement features at molecular absorption wavelengths (Stam et al. 2004; Stam 2008).

This paper is organized as follows. In Sect. 2, we formulate different types of errors in polarization degrees. Based on the derived equations, requirements for detection of a spectro-polarimetric feature are formulated and evaluated in Sect. 3. Then, we examine the feasibility of spectro-polarimetric 
searches for $\mathrm{H}_{2} \mathrm{O}$ vapor in Sect. 4. After discussions on unconsidered error factors and remaining future works in Sect. 5, we summarize and conclude this paper in Sect. 6.

\section{Formulation of errors}

We consider three types of errors: (a) errors arising from the difference between the efficiencies (throughputs) of two orthogonally polarized states, $b_{P}^{\text {eff }}$; (b) errors caused by speckle noises, $\sigma_{P}^{\text {spec }}$; and (c) errors arising from photon noise, $\sigma_{P}^{\text {phot }}$. (a) and (b) are systematic biases, whereas (c) is a random error. Each type of error is formulated below by expanding Eq. (1). Note that, in this section, we do not write out wavelength dependence in functions so as to avoid complicating the equations.

\subsection{Different efficiencies}

We analyze the effect of the difference between $r_{0}$ and $r_{90}$. By substituting $I^{\prime \mathrm{p}}$ in Eq. (2) into $I^{\mathrm{p}}$ in Eq. (1), the total error in the polarization degree is written as

$\mathcal{E}_{p} \cong b_{P}^{\mathrm{eff}}+\frac{\left(\epsilon_{0}-\epsilon_{90}\right)-\left(P+b_{P}^{\mathrm{eff}}\right)\left(\epsilon_{0}+\epsilon_{90}\right)}{2 \bar{r}_{\overline{\mathrm{I}}}}$

where

$$
\begin{aligned}
b_{P}^{\mathrm{eff}} & \equiv-\frac{1}{2}\left(\frac{r_{90}}{r_{0}}-1\right)\left(1-P^{2}\right) \\
& \cong\left(\frac{\delta \mathcal{S}}{\overline{\mathcal{S}}}+\frac{\delta \mathcal{T}}{\overline{\mathcal{T}}}+\frac{\delta k}{\bar{k}}\right)\left(1-P^{2}\right) .
\end{aligned}
$$

The derivation of the equations is given in Appendix A, where $\left|\epsilon_{\chi}\right| \ll r_{\chi} I_{\chi}^{\mathrm{p}}$ and $\left|r_{90} / r_{0}-1\right| \ll 1$ are assumed in the approximations. $b_{P}^{\text {eff }}$ is the bias caused by different efficiencies as it is caused by $r_{90} / r_{0}$. The barred values (e.g., $\bar{r}, \bar{I}$ ) satisfy $\bar{X}=\left(X_{0}+X_{90}\right) / 2$ and $\delta X$ (e.g., $\left.\delta \mathcal{S}, \delta \mathcal{T}\right)$ is defined by $\delta X=\left(X_{0}-X_{90}\right) / 2$. The terms $\delta \mathcal{S} / \overline{\mathcal{S}}, \delta \mathcal{T} / \overline{\mathcal{T}}$, and $\delta k / \bar{k}$ are the polarization degrees through interstellar, telluric, and instrumental (including telescope) transmission, respectively. The difference of total efficiencies, $r_{90} / r_{0}-1$, can be written as the sum of the three individual polarization terms.

Keller (1996) analyzed the effect of nonlinearity in detector responses. Following his formulation, this effect is implemented into $b_{P}^{\text {eff }}$ as

$b_{P}^{\mathrm{eff}} \cong\left(\frac{\delta \mathcal{S}}{\overline{\mathcal{S}}}+\frac{\delta \mathcal{T}}{\overline{\mathcal{T}}}+\frac{\delta k}{\bar{k}}\right)\left(1-P^{2}\right)\left(1+L \overline{I^{\mathrm{p}}}\right)$

where $L I^{\prime} \mathrm{p}$ is the nonlinearity term and has a typical value of $\sim 0.01$ (Keller 1996).

The second term in Eq. (4) is an error in $P$ caused by offset errors in intensity measurement. It can be either systematic or random: it is systematic if $\epsilon_{\chi}$ is systematic (e.g., due to speckle noises) and random if $\epsilon_{\chi}$ is random (e.g., due to photon noise). Each type of error is analyzed below.

\subsection{Speckle noises}

In high-contrast instruments, stellar intensity is reduced with a coronagraph. Even with an extreme AO system, a certain roughness of wavefront cannot be corrected, and hence, the speckle noises are generated on an image plane. The speckle noises disturb the detection and measurement of planetary photons. In this

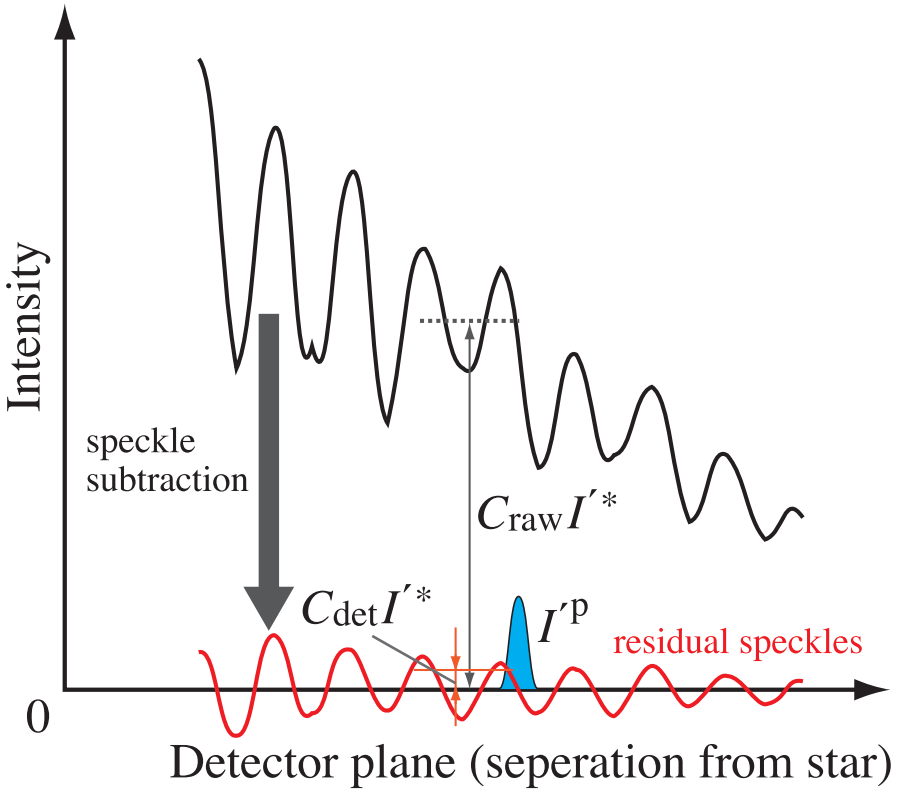

Fig. 2. Schematic illustration of an image profile before (upper line) and after (lower line) the speckle subtraction procedures. In the situation assumed for this paper, the speckle subtraction procedures include the SSDI but not the PDI at this stage. The PDI is conducted further on.

section, we examine the systematic bias in the planetary polarization degree due to the speckle noises.

We examine the second term of Eq. (4). In this paper, we consider a case where the PDI technique is applied after the SSDI procedure. The typical strength of $\epsilon_{\chi}$ (speckle noises) after the SSDI but before PDI is $C_{\operatorname{det}} I_{\chi}^{\prime *}\left(=C_{\operatorname{det}} r_{\chi} I_{\chi}^{*}\right)$, where $C_{\operatorname{det}}$ is the detection contrast of the instrument achieved with the SSDI but without the PDI. It describes the ratio of the strength of speckles to the non-coronagraphic stellar intensity, $I_{\chi}^{*}$ (see Fig. 2 for a schematic explanation and Appendix $\mathrm{C}$ for a more rigid description). Thus, we express

$\sigma_{\epsilon_{0}+\epsilon_{90}} \cong 2 C_{\mathrm{det}} \bar{r} \bar{I}^{*}$

$\sigma_{\epsilon_{0}-\epsilon_{90}} \cong g \cdot 2 C_{\mathrm{det}} \bar{r} \overline{I^{*}}$

where $\sigma_{\epsilon_{0}+\epsilon_{90}}$ and $\sigma_{\epsilon_{0}-\epsilon_{90}}$ represent the standard deviations of $\left(\epsilon_{0}+\epsilon_{90}\right)$ and $\left(\epsilon_{0}-\epsilon_{90}\right)$, respectively. Hence, $\left(\epsilon_{0}+\epsilon_{90}\right)$ and $\left(\epsilon_{0}-\epsilon_{90}\right)$ are typically within $\pm \sigma_{\epsilon_{0}+\epsilon_{90}}$ and $\pm \sigma_{\epsilon_{0}-\epsilon_{90}}$, respectively.

If both of the ordinary and extraordinary components of speckle noises have the same pattern and intensity, then we have $\epsilon_{0}-\epsilon_{90}=0$. However, it is not true due to, for example, different throughputs and wavefront errors for orthogonal polarized states; and polarization aberrations (deviation of the point-spread functions; Breckinridge \& Oppenheimer 2004; Breckinridge et al. 2015). Besides, it is not known yet how well the PDI after SSDI works because a spectro-polarimeter based on the combination of an IFS and a PBS has not been realized. Thus, we introduce a parameter, $g$, which expresses how well the PDI suppresses the speckle noise. The detection contrast improves from $C_{\text {det }}$ to $g C_{\text {det }}$ by applying the PDI. We consider the best and worst cases with regard to $g$. In the best case, $g$ is determined only by the difference in the throughputs, that is, $g=\delta r / \bar{r} \cong \delta k / \bar{k} \cong 0.05$. In the worst case, PDI does not work at all, that is, $g=1.0$.

A typical value of the error caused by the speckle noise $\left(\sigma_{P}^{\mathrm{spec}}\right)$ is derived from the second term of Eq. (4) using Eqs. (7)(8). If the spatial patterns of $\left(\epsilon_{0}-\epsilon_{90}\right)$ and $\left(\epsilon_{0}+\epsilon_{90}\right)$ are similar, 
we have

$\sigma_{P}^{\mathrm{spec}} \cong\left|g-\left(P+b_{P}^{\mathrm{eff}}\right)\right| \frac{C_{\mathrm{det}}}{C_{\mathrm{p} / \mathrm{s}}}$,

where $C_{\mathrm{p} / \mathrm{s}}=\overline{I^{\mathrm{p}}} / \overline{I^{*}}$ is the intensity contrast of a planet to its host star. Contrarily, if $\left(\epsilon_{0}-\epsilon_{90}\right)$ and $\left(\epsilon_{0}+\epsilon_{90}\right)$ are not correlated, we should express

$\sigma_{P}^{\mathrm{spec}} \cong \sqrt{g^{2}+\left(P+b_{P}^{\mathrm{eff}}\right)^{2}} \frac{C_{\mathrm{det}}}{C_{\mathrm{p} / \mathrm{s}}}$.

Because the highly correlated components between $\epsilon_{0}$ and $\epsilon_{90}$ will largely disappear in $\left(\epsilon_{0}-\epsilon_{90}\right)$, we expect that Eq. (10) is the case.

$\sigma_{P}^{\mathrm{spec}}$ is a function of $C_{\mathrm{det}} / C_{\mathrm{p} / \mathrm{s}}$. It is understandable that $\sigma_{P}^{\mathrm{spec}}$ is smaller when $C_{\text {det }}$ is better (smaller) and $C_{\mathrm{p} / \mathrm{s}}$ (the planetary brightness relative to the star) is greater. When $g \gg P+b_{P}^{\text {eff }}$, we have $\sigma_{P}^{\text {spec }} \cong g C_{\text {det }} / C_{\mathrm{p} / \mathrm{s}}$. This means that we have a smaller $\sigma_{P}^{\text {spec }}$ if we have a better (smaller) $g$, as expected. On the other hand, when $g \ll P+b_{P}^{\text {eff }}$, we have $\sigma_{P}^{\text {spec }} \cong\left(P+b_{P}^{\text {eff }}\right) C_{\text {det }} / C_{\mathrm{p} / \mathrm{s}}$. This indicates that, even if the PDI were perfect $(g=0), P$ would suffer from an error caused by the speckles, because we need to use not only a differential $\left(I^{\prime}{ }_{0}-I^{\prime}{ }_{90}\right)$ image but also a sum $\left(I^{\prime}{ }_{0}+I^{\prime}{ }_{90}\right)$ image to derive $P$.

It may be puzzling that we have a larger error when the planet is more polarized. This is a result of the fact that the change of a $P$ value due to virtually unpolarized speckle noises is greater when the planet is more polarized. Hence, most high-contrast polarimetry data is expressed in terms of polarized intensity, not degree of polarization. However, for ground-based spectropolarimetric detection of $\mathrm{H}_{2} \mathrm{O}$ (and other components common to Earth's atmosphere, e.g., $\mathrm{O}_{2}$ ), avoidance of deriving the degree of polarization (i.e., dividing by the sum data) may pose a problem because one must cancel out telluric features using a standard star spectrum, which will be observed at a different time and sky position from the target. Therefore, we continue to study the errors in degree of polarization, rather than polarized intensity.

As we assume the scaling factor at the head of Eq. (10) ranges from the order of $10^{-1}$ to unity, $C_{\mathrm{det}} / C_{\mathrm{p} / \mathrm{s}}$ should be less than the order of $10^{-1}$ to $10^{-2}$ so as to achieve a $\sigma_{P}^{\text {spec }}$ of a few percent.

\subsection{Photon noise}

If $\epsilon_{0}$ and $\epsilon_{90}$ in Eq. (2) are caused by photon noise and are thus random, the second term of Eq. (4) represents a random error $\left(\sigma_{P}^{\text {phot }}\right)$ in $P$. We consider scattered starlight remaining after a coronagraph as the dominating source of the photon noise. As the intensity of scattered starlight is $C_{\text {raw }} \bar{r} \bar{I}^{*}$, we have

$\sigma_{\epsilon_{0}+\epsilon_{90}} \cong \sigma_{\epsilon_{0}-\epsilon_{90}} \cong \sqrt{2 C_{\mathrm{raw}} \bar{r} \bar{I}^{*}}$.

Hence,

$$
\begin{aligned}
\sigma_{P}^{\mathrm{phot}} & \cong \frac{\sqrt{2\left(1+\left(P+b_{P}^{\mathrm{eff}}\right)^{2}\right) C_{\mathrm{raw}} \bar{r} \bar{I}^{*}}}{2 \bar{r} \overline{\mathrm{p}}} \cong \frac{\sqrt{2 C_{\mathrm{raw}} \bar{r} \bar{I}^{*}}}{2 \bar{r} \overline{\mathrm{p}}} \\
& =\frac{1}{\sqrt{2 \bar{r} \bar{I}^{*}}} \frac{\sqrt{C_{\mathrm{raw}}}}{C_{\mathrm{p} / \mathrm{s}}},
\end{aligned}
$$

where $\bar{I}^{*}$ is expressed in photon numbers. Naturally, the error from photon noise is smaller when the host star is brighter $\left(\bar{I}^{*}\right.$ is greater), the planet is brighter $\left(C_{\mathrm{p} / \mathrm{s}}\right.$ is greater $)$, and the instrument raw contrast is better ( $C_{\text {raw }}$ is less).

\subsection{Summary of error formulations}

In summary, the following types of errors in $P$ are formulated:

- Error from different efficiencies

$$
b_{P}^{\mathrm{eff}} \cong\left(\frac{\delta \mathcal{S}}{\overline{\mathcal{S}}}+\frac{\delta \mathcal{T}}{\overline{\mathcal{T}}}+\frac{\delta k}{\bar{k}}\right)\left(1-P^{2}\right)\left(1+L \overline{I^{\prime} \mathrm{p}}\right) \text {. }
$$

- Error from speckle noises

$$
\sigma_{P}^{\mathrm{spec}} \cong \sqrt{g^{2}+\left(P+b_{P}^{\mathrm{eff}}\right)^{2}} \frac{C_{\mathrm{det}}}{C_{\mathrm{p} / \mathrm{s}}} .
$$

- Error from photon noise

$$
\sigma_{P}^{\mathrm{phot}} \cong \frac{1}{\sqrt{2 \bar{r} \bar{I}^{*}}} \frac{\sqrt{C_{\mathrm{raw}}}}{C_{\mathrm{p} / \mathrm{s}}} .
$$

\section{Requirements for detecting a spectro-polarimetric feature}

In this section, requirements for detecting a spectro-polarimetric feature are formulated based on the derived equations for $b_{p}^{\text {eff }}$, $\sigma_{p}^{\text {spec }}$, and $\sigma_{P}^{\text {phot }}$ (Eqs. (13)-(15)). Then, we calculate these errors to obtain the requirements of the instruments.

\subsection{Different efficiencies}

A $b_{P}^{\text {eff }}$ spectrum has a spurious feature at the absorption wavelength $\lambda_{\mathrm{abs}}$, as described later. Therefore, the absolute strength of the spurious feature must be significantly smaller than that of the real feature on a $P$ spectrum for its detection. In other expressions, the following requirement should be satisfied:

$\left|\Delta b_{P}^{\mathrm{eff}}\right| \equiv\left|b_{P}^{\mathrm{eff}}\left(\lambda_{\mathrm{abs}}\right)-b_{P}^{\mathrm{eff}}\left(\lambda_{\mathrm{cnt}}\right)\right|<\omega \Delta P$,

where $\Delta P\left(\equiv P\left(\lambda_{\mathrm{abs}}\right)-P\left(\lambda_{\mathrm{cnt}}\right)\right)$ is the polarization degree of the feature and $w$ is a coefficient determined by the detection definition. $w$ is set to $1 / 3$ throughout the paper.

We verify that the $b_{P}^{\text {eff }}$ spectrum has a feature at $\lambda_{\text {abs }}$ by examining the three factors in Eq. (13). The following properties are found:

- The first factor, $(\delta \mathcal{S} / \overline{\mathcal{S}}+\delta \mathcal{T} / \overline{\mathcal{T}}+\delta k / \bar{k})$, is presumably featureless. The reasons are given in the following paragraph.

- The second factor, $\left(1-P^{2}\right)$, has a feature if the $P$ spectrum has a real feature. If $P$ does not have a feature, neither does $\left(1-P^{2}\right)$, which is quite evident.

- The third factor, $\left(1+L I^{\overline{\mathrm{p}}}\right)$, has a feature, even if $I^{\mathrm{p}}$ (and thus $P$ ) does not have a feature. This is because the detector nonlinearity term, $L I^{\overline{\mathrm{p}}}$, is proportional to $\bar{I}^{\overline{\mathrm{p}}}\left(=\overline{\mathcal{S}} \overline{\mathcal{T}} \bar{k} \overline{I^{\mathrm{p}}}\right)$ and telluric transmittance, $\overline{\mathcal{T}}$, always has an $\mathrm{H}_{2} \mathrm{O}$ absorption feature at $\lambda_{\mathrm{abs}}$.

At the end, the spectrum of $b_{P}^{\text {eff }}$ will have a spurious feature at $\lambda_{\text {abs }}$. Therefore, the requirement Eq. (16) must be satisfied.

We describe the reasons why $(\delta \mathcal{S} / \overline{\mathcal{S}}+\delta \mathcal{T} / \overline{\mathcal{T}}+\delta k / \bar{k})$ is presumed to be featureless by looking at each term separately. Although $\mathcal{S}$ may have a weak feature at $\lambda_{\text {abs }}$, interstellar polarization $\delta \mathcal{S} / \overline{\mathcal{S}}$ does not have a feature at $\lambda_{\text {abs }}$ because the polarization is caused by aligned dust particles and is not due to $\mathrm{H}_{2} \mathrm{O}$ molecules (see $P$ spectra of polarized standard stars, which are polarized through interstellar transmission, e.g., Fossati et al. 2007). $\mathcal{T}$ has a strong feature at $\lambda_{\mathrm{abs}}$. However, similar to the 
Table 1. Input values to the calculations.

\begin{tabular}{|c|c|c|c|}
\hline Parameter & Description & Values & References, notes \\
\hline $\begin{array}{l}P\left(\lambda_{\mathrm{cnt}}\right) \\
\Delta P \\
\lambda_{\mathrm{abs}} \\
\Delta \lambda \\
{ }^{\dagger} p\left(\lambda_{\mathrm{cnt}}\right) \\
{ }^{\dagger} \mu \\
{ }^{\dagger} R_{\mathrm{p}} \\
{ }^{\dagger} a_{\mathrm{p}} \\
\dagger\end{array}$ & $\begin{array}{l}\text { - Planet - } \\
\text { pol. deg. at continuum } \\
\text { pol. enhancement at feature }\left(\mathrm{H}_{2} \mathrm{O}\right) \\
\text { absorption wavelength }\left(\mathrm{H}_{2} \mathrm{O}\right) \\
\text { wavelength width of feature }\left(\mathrm{H}_{2} \mathrm{O}\right) \\
\text { geometric albedo } \\
\text { abs. strength, }\left[p\left(\lambda_{\mathrm{cnt}}\right)-p\left(\lambda_{\mathrm{abs}}\right)\right] / p\left(\lambda_{\mathrm{cnt}}\right) \\
\text { planet radius } \\
\text { orbital distance } \\
\text { phase angle }\end{array}$ & $\begin{array}{r}0.1 \\
0.1 \\
1.12 \mu \mathrm{m} \\
0.05 \mu \mathrm{m} \\
0.25 \\
0.5 \\
\text { var } \\
\text { var } \\
90^{\circ}\end{array}$ & $\begin{array}{l}\text { Miles-Páez et al. (2014) } \\
\text { Miles-Páez et al. (2014) } \\
\text { Miles-Páez et al. (2014) } \\
\text { Miles-Páez et al. (2014) } \\
\text { Montañés-Rodriguez et al. (2005) } \\
\text { Turnbull et al. (2006) } \\
\text { Exoplanet.eu }{ }^{\ddagger} \text { or estimated from mass* } \\
\text { Exoplanet.eu }{ }^{\ddagger}\end{array}$ \\
\hline $\begin{array}{l}m^{*} \\
\dagger d\end{array}$ & $\begin{array}{l}\text { - Host star - } \\
\text { stellar magnitude in } J \text { band (Fig. 4) } \\
\text { (Table } 2 \text { and Fig. 6) } \\
\text { distance from Earth }\end{array}$ & $\begin{array}{r}0,5,10 \mathrm{mag} \\
\text { var } \\
\text { var }\end{array}$ & $\begin{array}{l}\text { SIMBAD }^{* *} \\
\text { Exoplanet.eu }{ }^{\ddagger} \text { or SIMBAD** }\end{array}$ \\
\hline $\begin{array}{l}\delta \mathcal{S} / \overline{\mathcal{S}} \\
\delta \mathcal{T} / \overline{\mathcal{T}} \\
\mathcal{T}\left(\lambda_{\mathrm{abs}}\right) \\
\mathcal{T}\left(\lambda_{\mathrm{cnt}}\right)\end{array}$ & $\begin{array}{l}\text { - Interstellar, telluric - } \\
\text { interstellar polarization } \\
\text { pol. through telluric transmission } \\
\text { telluric transmittance at } \lambda_{\mathrm{abs}} \\
\text { telluric transmittance at } \lambda_{\mathrm{cnt}}\end{array}$ & $\begin{array}{r}0.01 \\
5.0 \times 10^{-5} \\
0.7 \\
0.99\end{array}$ & $\begin{array}{l}\text { Bailey et al. ( } 2008) \\
\text { Alt. } \sim 3000 \mathrm{~m} \text {. Based on GEMINI-S data } \\
\text { Same as } \mathcal{T}\left(\lambda_{\text {abs }}\right) .\end{array}$ \\
\hline $\begin{array}{l}\Delta t \\
D \\
\bar{k} \\
\delta k / \bar{k} \\
L I^{\prime \mathrm{p}}\left(\lambda_{\text {cnt }}\right) \\
{ }^{\dagger} C_{\text {raw }} \\
{ }^{\dagger} C_{\text {det }} \\
w\end{array}$ & $\begin{array}{l}\text { - Observation, instrument - } \\
\text { integration time } \\
\text { diameter of the telescope mirror } \\
\text { averaged efficiency: }\left(k_{0}+k_{90}\right) / 2 \\
\text { instrumental (inc. telescope) pol. } \\
\text { detector nonlinearity term } \\
\text { inst. raw contrast } \\
\text { inst. detection contrast before PDI }(1 \sigma) \\
\text { detection criteria definition }\end{array}$ & $\begin{array}{r}15 \mathrm{~h} \\
39 \mathrm{~m} \\
0.1 \\
0.05 \\
0.01 \\
\text { (see Fig. } 6) \\
\text { (see Fig. } 6 \text { ) } \\
1 / 3\end{array}$ & $\begin{array}{l}\text { E-ELT } \\
\text { EPOL goal, Keller et al. (2010) } \\
\text { E-ELT, de Juan Ovelar et al. (2012) } \\
\text { Keller (1996) } \\
\text { EPICS, Fig. } 3 \text { of Kasper et al. (2010) } \\
\text { EPICS-IFS, Fig. } 4 \text { of Kasper et al. (2010) }\end{array}$ \\
\hline
\end{tabular}

Notes. ${ }^{(\dagger)}$ Not used in the calculations for Figs. 3, 4, but used for Table 2 and Fig. 6. (亡) The Extrasolar Planets Encyclopaedia, http: //exoplanet. eu/, Schneider et al. (2011). ${ }^{(*)}$ Using the mass-radius relations in Weiss et al. (2013) and Weiss \& Marcy (2014). See text for details. ${ }^{(* *)}$ http://simbad.u-strasbg.fr/simbad/, Wenger et al. (2000). ${ }^{(\S)}$ http://www. gemini.edu/sciops/telescopes-and-sites/ observing-condition-constraints/ir-transmission-spectra, Lord (1992).

case of $\delta \mathcal{S} / \overline{\mathcal{S}}$, telluric polarization $\delta \mathcal{T} / \overline{\mathcal{T}}$ should be featureless at $\lambda_{\text {abs }}$ because the polarization is produced by dust particles (Bailey et al. 2008). Since $k$ is determined by optical elements, $k$ and $\delta k / \bar{k}$ do not have a feature at $\lambda_{\text {abs. }}$. Thus, we regard the spectrum of $(\delta \mathcal{S} / \overline{\mathcal{S}}+\delta \mathcal{T} / \overline{\mathcal{T}}+\delta k / \bar{k})$ as featureless.

The value of $\left|\Delta b_{P}^{\text {eff }}\right|$ is calculated using Eq. (13) and the values listed in Table 1. As a result, we obtain $\left|\Delta b_{P}^{\text {eff }}\right| \cong 2.2 \times 10^{-3}$, which satisfies the requirement of Eq. (16).

Here we address the $\delta \mathcal{S} / \overline{\mathcal{S}}, \delta \mathcal{T} / \overline{\mathcal{T}}$, and $\delta k / \bar{k}$ values, which are used for the calculation. All of them are wavelengthdependent. However, in order to simplify the calculation, we treat $\delta \mathcal{S} / \overline{\mathcal{S}}, \delta \mathcal{T} / \overline{\mathcal{T}}$, and $\delta k / \bar{k}$ as constants within a minimum wavelength range necessary to detect a feature, that is, the range between $\lambda_{\mathrm{abs}} \pm \Delta \lambda(\Delta \lambda$ is the FWHM of the feature, $\sim 0.05 \mu \mathrm{m})$. This assumption is allowed because they are expected to be featureless, as described above.

The interstellar polarization $(\delta \mathcal{S} / \overline{\mathcal{S}})$ is set to be $1 \%$ as a safe assumption. Note that most strongly polarized standard stars, which are polarized through a relatively long (a few hundred par$\mathrm{sec}$ ) interstellar path, have a polarization degree of one to a few percent (Whittet et al. 1992). As direct observation of exoplanets is limited to relatively nearby systems (within $\sim 30 \mathrm{pc}$ ), we expect that the interstellar polarization for many of the targets is likely to be lower than the assumed value. As for polarization through telluric transmission $(\delta \mathcal{T} / \overline{\mathcal{T}})$, we take a value $5.0 \times 10^{-5}$, measured by Bailey et al. (2008). We assume instrumental (including telescope) polarization $(\delta k / \bar{k})$ to be $5 \%$, based on modeling for the E-ELT by de Juan Ovelar et al. (2012). We do not know the signatures (position angles of polarization) of $\delta \mathcal{S} / \overline{\mathcal{S}}$, $\delta \mathcal{T} / \overline{\mathcal{T}}$, and $\delta k / \bar{k}$, but assume all of them to be positive so as to maximize the value of their sum.

For the purpose of understanding how $\left|\Delta b_{P}^{\mathrm{eff}}\right|$ is determined, it is written as

$$
\begin{aligned}
\left|\Delta b_{P}^{\mathrm{eff}}\right| \cong & |\delta \mathcal{S} / \overline{\mathcal{S}}+\delta \mathcal{T} / \overline{\mathcal{T}}+\delta k / \bar{k}| \\
& \times\left|\left(1-P\left(\lambda_{\mathrm{cnt}}\right)^{2}\right)\left(\Delta\left[L \overline{I^{\prime} \mathrm{p}}\right]\right)-\left(1+L \overline{I^{\prime} \mathrm{p}}\left(\lambda_{\mathrm{cnt}}\right)\right) \Delta\left[P^{2}\right]\right| \\
\cong & |\delta \mathcal{S} / \overline{\mathcal{S}}+\delta \mathcal{T} / \overline{\mathcal{T}}+\delta k / \bar{k}| \times\left|\Delta\left[L \overline{I^{\mathrm{p}}}\right]-\Delta\left[P^{2}\right]\right| \\
\cong & \left|0.01+5.0 \times 10^{-5}+0.05\right| \times|-0.0065-0.03|,(17)
\end{aligned}
$$

where $\Delta[X]$ represents $X\left(\lambda_{\mathrm{abs}}\right)-X\left(\lambda_{\mathrm{cnt}}\right)$. It is seen that the contribution from $\delta \mathcal{T} / \overline{\mathcal{T}}$ is negligible. We also find that the ratio of contributions from the $L I^{\overline{\mathrm{p}}}$ and $P$ is approximately $1: 5$. 

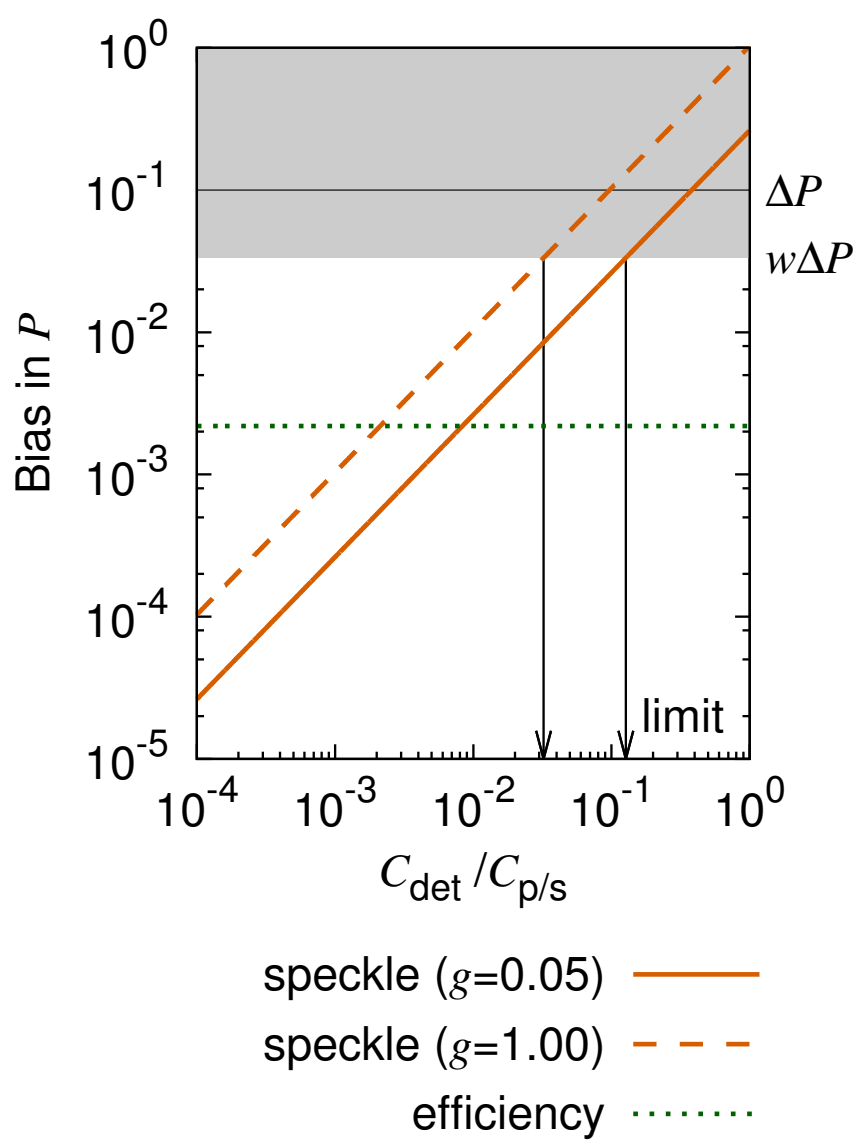

Fig. 3. Bias in $P$ with respect to $C_{\text {det }} / C_{\mathrm{p} / \mathrm{s}}\left(\lambda_{\mathrm{abs}}\right)$. The speckle error $\sigma_{P}^{\text {spec }}\left(\lambda_{\mathrm{abs}}\right)$ for $g=0.05$ (solid line) and $g=1.0$ (dashed line) are plotted. The efficiency error $\left|\Delta b_{p}^{\text {eff }}\right|$ (dotted line) is also shown. The shaded areas show where detection is difficult because the bias is larger than $w \Delta P$. The arrows point to the maximum limit of $C_{\mathrm{det}} / C_{\mathrm{p} / \mathrm{s}}\left(\lambda_{\mathrm{abs}}\right)$ for the feature detection. $w$ is set to one third.

\subsection{Speckle noises}

Speckles move outward from the host star on an image plane as the wavelength increases (see Fig. 23 in Sparks \& Ford 2002), whereas the position of a planet image is independent of the wavelength. The SSDI technique removes the speckles using this property; however, some residuals will remain. Because the speckles "cross" a planet on a space of the separation $(\theta)$ and the wavelength ( $\lambda$; see Fig. 2 in Rodenhuis et al. 2012), they will appear as spurious features on the $I^{\prime \mathrm{p}}$ and $P$ spectra. It is difficult to distinguish the spurious speckle features from the real molecular feature based on their spectral widths (see Appendix B). Therefore, it must be guaranteed that the strength of the speckle features on the $P$ spectrum is sufficiently small compared to that of the real feature, $\Delta P$.

Unlike the efficiency error, $b_{P}^{\text {eff }}$, we only know the standard deviation of the speckle error, $\sigma_{P}^{\text {spec }}$. Thus, we need to define the detection requirement differently: both $\sigma_{P}^{\text {spec }}\left(\lambda_{\text {abs }}\right)$ and $\sigma_{P}^{\text {spec }}\left(\lambda_{\text {cnt }}\right)$ must be significantly smaller than $\Delta P$. Owing to a greater $P$ and a smaller $C_{\mathrm{p} / \mathrm{s}}$ at $\lambda_{\mathrm{abs}}$ than those at $\lambda_{\mathrm{cnt}}$, it is expected that $\sigma_{P}^{\text {spec }}\left(\lambda_{\text {abs }}\right)>\sigma_{P}^{\text {spec }}\left(\lambda_{\text {cnt }}\right)$, assuming Eq. (16) is satisfied (see Eq. (14)). Hence, the requirement regarding $b_{P}^{\text {spec }}$ is expressed as

$\sigma_{P}^{\mathrm{spec}}\left(\lambda_{\mathrm{abs}}\right)<w \Delta P$.

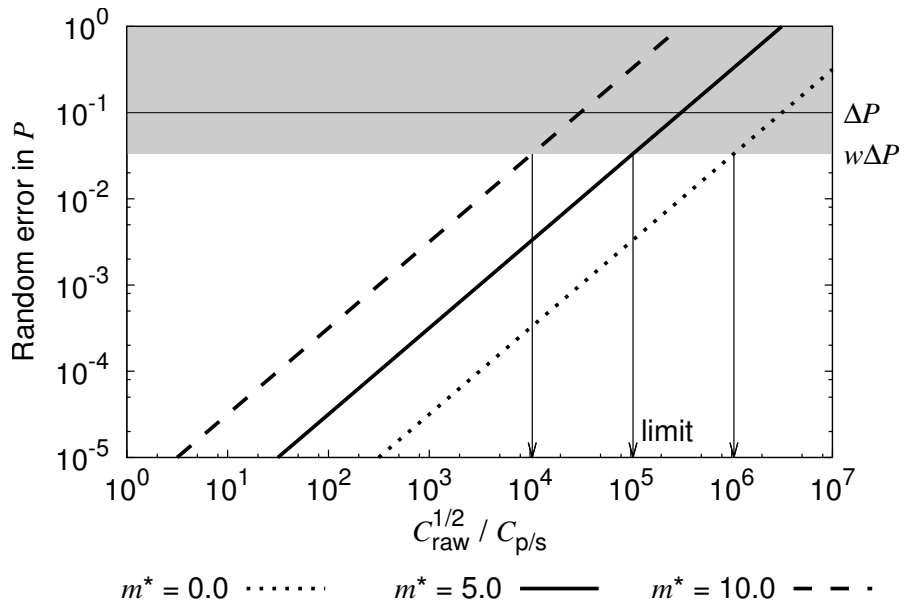

Fig. 4. $\sigma_{P}^{\text {phot }}\left(\lambda_{\text {abs }}\right)$ with respect to $\sqrt{C_{\text {raw }}} / C_{\mathrm{p} / \mathrm{s}}\left(\lambda_{\mathrm{abs}}\right)$ for different stellar magnitudes: $m^{*}=0.0$ (dotted line), 5.0 (solid line), 10.0 (dashed line). Magnitude $m^{*}$ determines $\bar{I}^{*}{ }_{\chi}$ in Eq. (15). The shaded area shows where detection is difficult because $\sigma_{P}^{\text {phot }}$ is larger than $w \Delta P$. The arrows point to the maximum limit of $\sqrt{C_{\text {raw }}} / C_{\mathrm{p} / \mathrm{s}}\left(\lambda_{\mathrm{abs}}\right)$ for detection. $w$ is set to one third.

Using Eq. (14), the values of $\sigma_{P}^{\text {spec }}\left(\lambda_{\text {abs }}\right)$ are calculated as a function of $C_{\mathrm{det}} / C_{\mathrm{p} / \mathrm{s}}\left(\lambda_{\mathrm{abs}}\right)$. As described, we consider two cases with regard to $g: g=0.05$ as the best case and $g=1.0$ as the worst. All the other input values are given in Table 1.

Figure 3 displays $\sigma_{P}^{\text {spec }}\left(\lambda_{\text {abs }}\right)$ together with $\left|\Delta b_{P}^{\text {eff }}\right|$. As shown in Eq. (14), $\sigma_{P}^{\text {spec }}\left(\lambda_{\mathrm{abs}}\right)$ is linearly correlated to $C_{\mathrm{det}} / C_{\mathrm{p} / \mathrm{s}}\left(\lambda_{\mathrm{abs}}\right)$. For the best case $(g=0.05), C_{\text {det }}$ should be better than $\sim 1.3 \times$ $10^{-1} C_{\mathrm{p} / \mathrm{s}}\left(\lambda_{\mathrm{abs}}\right)$, which means that a contrast performance (before PDI) to achieve $\sim 8$-sigma detection of the planet signal is required. For the worst case $(g=1.0), C_{\text {det }}$ should be better than $\sim 3.2 \times 10^{-2} C_{\mathrm{p} / \mathrm{s}}\left(\lambda_{\mathrm{abs}}\right)$, meaning a requirement of $\sim 30$-sigma detection performance. Naturally, the requirement for $C_{\text {det }}$ is less strict for the case of a better $g$.

The detectability is limited by the speckle error $\sigma_{P}^{\text {spec }}\left(\lambda_{\text {abs }}\right)$ rather than the efficiency error $\left|\Delta b_{P}^{\text {eff }}\right|$ because $\left|\Delta b_{P}^{\text {eff }}\right|$ is small enough compared to $\Delta P$. Thus, hereafter, the requirement pertaining to the efficiency error (Eq. (16)) is neglected in the evaluation of the detectability, though $b_{P}^{\text {eff }}$ in Eq. (14) is still taken into account.

\section{3. photon noise}

The error due to photon noise $\sigma_{P}^{\text {phot }}$ should be smaller than $\Delta P$ both at $\lambda_{\mathrm{cnt}}$ and $\lambda_{\mathrm{abs}}$. Because of the smaller $\bar{r} \bar{I}^{*}$ and $C_{\mathrm{p} / \mathrm{s}}$ at $\lambda_{\mathrm{abs}}$ than at $\lambda_{\text {cnt }}$, we have $\sigma_{P}^{\text {phot }}\left(\lambda_{\text {abs }}\right)>\sigma_{P}^{\text {phot }}\left(\lambda_{\text {cnt }}\right)$. Thus, the requirement regarding the photon noise is written as

$\sigma_{P}^{\text {phot }}\left(\lambda_{\mathrm{abs}}\right)<w \Delta P$

Using Eq. (15), the values of $\sigma_{P}^{\text {phot }}\left(\lambda_{\mathrm{abs}}\right)$ are calculated as a function of $\sqrt{C_{\text {raw }}} / C_{\mathrm{p} / \mathrm{s}}\left(\lambda_{\mathrm{abs}}\right)$ for stellar magnitudes $m^{*}=0.0,5.0$, and 10.0 (Fig. 4). Telescope diameter $D=39 \mathrm{~m}$ (E-ELT) and total integration time $\Delta t=15 \mathrm{~h}(5 \mathrm{~h} \times 3$ nights $)$ are taken to derive the stellar photon counts $2 \bar{r} \bar{I}^{*}$. The other settings are summarized in Table 1.

As seen in Fig. 4, for a host star of magnitude $m^{*}=5.0$, $\sqrt{C_{\text {raw }}} / C_{\mathrm{p} / \mathrm{s}}\left(\lambda_{\mathrm{abs}}\right)$ should be smaller than $\sim 1 \times 10^{5}$. In other words, $C_{\text {raw }}$ should be smaller than $\sim 1 \times 10^{10}\left(C_{\mathrm{p} / \mathrm{s}}\left(\lambda_{\mathrm{abs}}\right)\right)^{2}$. As might be 
expected, the requirement for $C_{\text {raw }}$ is less strict if the host star is brighter.

\section{Evaluation of feasibility}

\subsection{Expected number of observable planets}

We discuss the feasibility of spectro-polarimetry through estimating the number of planets for which a $\mathrm{H}_{2} \mathrm{O}$ feature with $P\left(\lambda_{\text {cnt }}\right)=10 \%$ and $\Delta P=10 \%$ is detectable. The detectability is estimated according to Eqs. (18) and (19) by calculating $\sigma_{P}^{\text {spec }}\left(\lambda_{\text {abs }}\right)$ and $\sigma_{P}^{\text {phot }}\left(\lambda_{\text {abs }}\right)$ for the 2041 discovered planets listed in the Exoplanet.eu (the Extrasolar Planets Encyclopaedia, Schneider et al. 2011) database as of January 7, 2016. Assuming we use the E-ELT at Cerro Armazones, Chile, we exclude objects with declination $>+35^{\circ}$.

In Eq. (15) $C_{\text {raw }}$ is taken from the values delivered by the AO system (called XAO) for EPICS, a planned direct observing instrument for exoplanets (Fig. 3 in Kasper et al. 2010). We choose EPICS-IFS as the reference of $C_{\text {det }}$ in Eq. (14) (Fig. 4 in Kasper et al. 2010), because: (1) it is actually being studied for the E-ELT; (2) it covers the $\lambda_{\mathrm{abs}}(=1.12 \mu \mathrm{m})$; and (3) its $C_{\mathrm{det}}$ can be applied directly to our formulae because the values without the PDI procedure are provided in Kasper et al. (2010). Note that EPICS-IFS itself will not have a spectro-polarimetry capability. We consider a hypothetical spectro-polarimeter with a contrast performance (before PDI) comparable to that of EPICS-IFS. The $C_{\text {det }}$ values of EPICS-IFS are converted from 5-sigma to 1-sigma values to fit our formulation because we use an external parameter $w$ to define the criteria of the detection.

In Eqs. (14) and (15), $C_{\mathrm{p} / \mathrm{s}}$ for each planet is calculated by

$C_{\mathrm{p} / \mathrm{s}}(\lambda)=p(\lambda) \Phi_{\alpha}\left[\frac{R_{\mathrm{p}}}{a_{\mathrm{p}}}\right]^{2}=p(\lambda) \Phi_{\alpha}\left[\frac{R_{\mathrm{p}}}{d \tan \left(\theta_{\mathrm{p}}\right)}\right]^{2}$,

$$
\Phi_{\alpha}=[\sin (\alpha)+(\pi-\alpha) \cos (\alpha)] / \pi,
$$

where $p(\lambda)$ is the geometric albedo of a planet at wavelength $\lambda, \Phi_{\alpha}$ is the phase law at phase angle $\alpha$ for a Lambert sphere, $R_{\mathrm{p}}$ is the planet radius, $a_{\mathrm{p}}$ is the distance from the host star to the planet, and $d$ is the distance to the star from an observer (Traub \& Oppenheimer 2010). $p(\lambda)$ and $\alpha$ are fixed for all the planets (Table 1). $R_{\mathrm{p}}, a_{\mathrm{p}}$ (assumed to be close to the semimajor axis), and $d$ is obtained primarily from the Exoplanet.eu database. If $d$ is not available, we transform it from the parallax data in the SIMBAD database (Wenger et al. 2000). If $R_{\mathrm{p}}$ is not found in the Exoplanet.eu database, we predict it based on the empirical mass-radius relations studied by Weiss et al. (2013) and Weiss \& Marcy (2014), where the planet mass $\left(M_{\mathrm{p}}\right)$ is acquired from the Exoplanet.eu. Different relations are applicable, depending on the different ranges of the planet mass $\left(M_{\mathrm{p}}\right)$ : (1) $M_{\mathrm{p}}>150 M_{\text {Earth }}$; (2) $10 M_{\text {Earth }}<M_{\mathrm{p}} \leq 150 M_{\text {Earth }}$ (Weiss et al. 2013); (3) $4 M_{\text {Earth }}<M_{\mathrm{p}} \leq 10 M_{\text {Earth }}$, and (4) $M_{\mathrm{p}}<$ $4 M_{\text {Earth }}$ (Weiss \& Marcy 2014). Weiss et al. (2013) show that the radius is weakly (exponents -0.03 or 0.094 ) correlated with the incident stellar flux on the planet for the $M_{\mathrm{p}}$ ranges of (1) and (2). The time-averaged incident flux is calculated using the stellar effective temperature, the stellar radius, the semi-major axis, and the orbital eccentricity, which are retrieved primarily from the Exoplanet.eu database. If the eccentricity is not provided in the database, it is assumed to be zero. If either the stellar temperature or radius is not in the database, we use the mean flux in Weiss et al. $\left(2013 ; 8.6 \times 10^{8} \mathrm{erg} \mathrm{s}^{-1} \mathrm{~cm}^{-2}\right)$.

The SIMBAD database (Wenger et al. 2000) is used for the reference of the $J$-band magnitudes $(m *)$ of the host stars, which

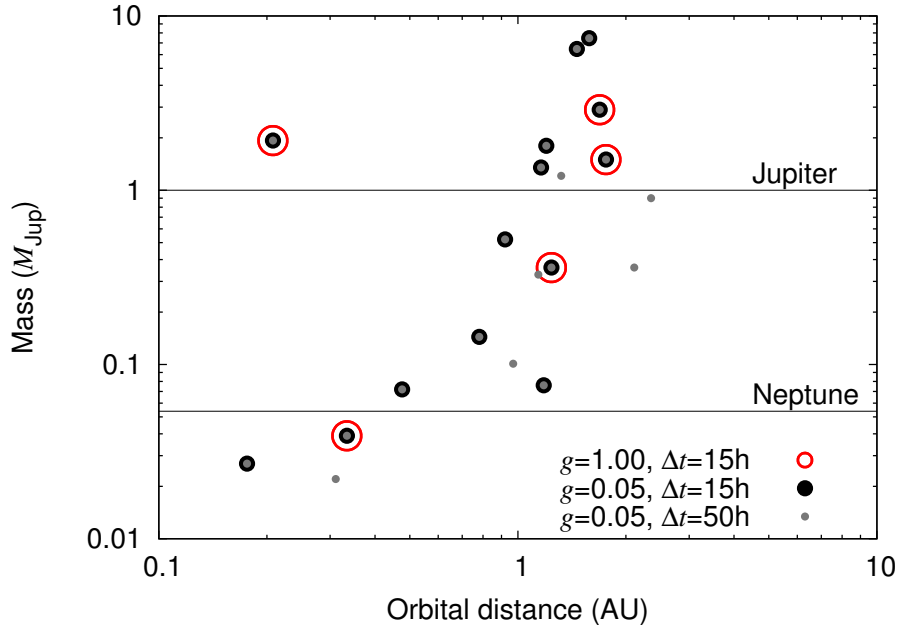

Fig. 5. Mass and orbital distances of the observable planets. The open circles are planets observable with a setting of $g=1.0$ and $\Delta t=15 \mathrm{~h}$. The filled larger circles are those with a setting of $g=0.05$ and $\Delta t=$ $15 \mathrm{~h}$. The filled smaller circles are those with a setting of $g=0.05$ and $\Delta t=50 \mathrm{~h}$.

determine $\overline{I_{\gamma}^{*}}\left(\lambda_{\mathrm{abs}}\right)$ in Eq. (15). All the other fixed input values to the calculations are shown in Table 1.

Table 2 lists the planets for which we predict the spectropolarimetric detection of the $\mathrm{H}_{2} \mathrm{O}$ feature is possible with the setting $g=0.05$. We have extracted 14 planets, of which 11 are estimated to be located within the snow line. The snow line distance is calculated according to Eq. (17) in Ida \& Lin (2005). Among the 14 observable planets with $g=0.05$, five planets are observable even with $g=1.0$. Figure 5 shows the mass and orbital distances of the observable planets. The mass of the planets range from $7.5 M_{\mathrm{Jup}}\left(\mathrm{HD} 60532\right.$ c) to $0.027 M_{\mathrm{Jup}} \cong 0.5 M_{\mathrm{Nep}}$ (GJ 682 c).

In order to illustrate our results graphically, we transform the expressions for the detection requirements (Eqs. (18) and (19)) into suitable forms:

$$
\begin{aligned}
& \frac{1}{w} \sqrt{g^{2}+\left(P\left(\lambda_{\mathrm{abs}}\right)+b_{P}^{\mathrm{eff}}\left(\lambda_{\mathrm{abs}}\right)\right)^{2}} C_{\mathrm{det}}<\Delta P C_{\mathrm{p} / \mathrm{s}}\left(\lambda_{\mathrm{abs}}\right), \\
& \frac{1}{w} \frac{1}{\sqrt{2 \bar{r}\left(\lambda_{\mathrm{abs}}\right) \bar{I}^{*}\left(\lambda_{\mathrm{abs}}\right)}} \sqrt{C_{\mathrm{raw}}}<\Delta P C_{\mathrm{p} / \mathrm{s}}\left(\lambda_{\mathrm{abs}}\right) .
\end{aligned}
$$

The right-hand side of the inequations above is interpreted as the contrast of the spectro-polarimetric feature signals to the host star signals. The left hand side of Eqs. (22) and (23) are contrast performances for the feature detection, constrained by the speckle noises and the photon noise, respectively.

In Fig. 6, we plot the (i) speckle-limited contrast (the lefthand side of Eq. (22)); (ii) photon-limited contrast (the left-hand side of Eq. (23)); and (iii) spectro-polarimetric feature contrast $\left(\triangle P C_{\mathrm{p} / \mathrm{s}}\right)$ against the separation from the star. When both lines for (i) and (ii) are below (iii) in the figure, the feature is detectable. Four different cases are shown: (a) a "typical" planet with averaged properties of the observable planets with $g=1.0$; (b) same as (a) except for $g=0.05$. (c) Gliese 876 b with $g=1.0$; and (d) Gliese $876 \mathrm{~b}$ with $g=0.05$. Except for (a), detectability is determined by the photon-limited contrast for a certain $\theta$ range. Thus, the detectability can be improved by obtaining more photons (e.g., with a longer $\Delta t$ or a better $\bar{k}$ ). In fact, if we extend 
Table 2. List of planets for which spectro-polarimetric detection of $\mathrm{H}_{2} \mathrm{O}$ vapor is possible with $g=0.05$.

\begin{tabular}{|c|c|c|c|c|c|c|c|c|c|c|c|c|}
\hline Planet & $\begin{array}{l}m^{*}(J) \\
(\mathrm{mag}) \\
\end{array}$ & $\begin{array}{c}d \\
(\mathrm{pc})\end{array}$ & Sp. type & $\begin{array}{c}M_{\mathrm{p}} \\
\left(M_{\mathrm{Jup}}\right) \\
\end{array}$ & $\begin{array}{c}R_{\mathrm{p}}^{\dagger} \\
\left(10^{4} \mathrm{~km}\right) \\
\end{array}$ & $\begin{array}{c}\theta_{\mathrm{p}} \\
\left({ }^{\prime \prime}\right)\end{array}$ & $\begin{array}{c}a_{\mathrm{p}} \\
(\mathrm{AU})\end{array}$ & $\mathrm{SL}^{\ddagger}$ & $\begin{array}{c}\sigma_{P}^{\text {phot }} \\
(\%) \\
\end{array}$ & $\frac{\Delta P}{\sigma_{P}^{\text {phot }}}$ & $\begin{array}{c}\sigma_{P}^{\mathrm{spec}} \\
(\%) \\
\end{array}$ & $\frac{\Delta P}{\sigma_{P}^{\text {spec }}}$ \\
\hline $55 \mathrm{Cnc} f$ & 4.6 & 12.3 & K0IV-V & $1.4 \mathrm{E}-01$ & $5.6(2)$ & 0.06 & 0.78 & in & 1.9 & 5 & 1.3 & 8 \\
\hline 61 Vir d & 3.3 & 8.5 & G5V & $7.2 \mathrm{E}-02$ & $3.8(2)$ & 0.06 & 0.48 & in & 1.0 & 10 & 1.6 & 6 \\
\hline Aldebaran b & -2.1 & 20.4 & K5III & $6.5 \mathrm{E}+00$ & $7.3(1)$ & 0.07 & 1.46 & in & 0.2 & 62 & 1.6 & 6 \\
\hline GJ 682 c & 6.5 & 5.1 & $\mathrm{M} 3.5 \mathrm{~V}$ & $2.7 \mathrm{E}-02$ & $2.3(3)$ & 0.04 & 0.18 & in & 2.9 & 4 & 2.3 & 4 \\
\hline$\S$ Gliese 876 b & 5.9 & 4.7 & M4V & $1.9 \mathrm{E}+00$ & $4.1(1)$ & 0.04 & 0.21 & in & 0.7 & 15 & 0.6 & 18 \\
\hline$\S$ Gliese 876 e & 5.9 & 4.7 & M4V & $3.9 \mathrm{E}-02$ & $3.0(2)$ & 0.07 & 0.33 & out & 2.0 & 5 & 0.5 & 19 \\
\hline$\S^{\S} \mathrm{HD} 113538 \mathrm{~b}$ & 6.4 & 15.8 & K9V & $3.6 \mathrm{E}-01$ & $9.9(2)$ & 0.08 & 1.24 & out & 2.9 & 3 & 0.5 & 20 \\
\hline$\S^{\S} \mathrm{HD} 176051 \mathrm{~b}$ & 3.9 & 15.0 & & $1.5 \mathrm{E}+00$ & $8.5(1)$ & 0.12 & 1.76 & $?$ & 1.7 & 6 & 0.8 & 13 \\
\hline HD $192310 \mathrm{c}$ & 4.1 & 8.8 & $\mathrm{~K} 3 \mathrm{~V}$ & $7.6 \mathrm{E}-02$ & $4.2(2)$ & 0.13 & 1.18 & in & 3.2 & 3 & 1.0 & 10 \\
\hline HD $27442 \mathrm{~b}$ & 2.6 & 18.1 & $\mathrm{~K} 2 \mathrm{IVa}$ & $1.4 \mathrm{E}+00$ & $6.0(1)$ & 0.06 & 1.16 & in & 1.4 & 7 & 2.3 & 4 \\
\hline HD $60532 \mathrm{c}$ & 3.7 & 25.7 & F6IV-V & $7.5 \mathrm{E}+00$ & $8.0(1)$ & 0.06 & 1.58 & in & 2.6 & 4 & 3.0 & 3 \\
\hline$\S \mathrm{HD} 62509 \mathrm{~b}$ & -0.5 & 10.3 & KOIIIb & $2.9 \mathrm{E}+00$ & $5.7(1)$ & 0.16 & 1.69 & in & 0.4 & 28 & 0.8 & 12 \\
\hline$\alpha$ Ari b & 0.1 & 20.2 & K2III & $1.8 \mathrm{E}+00$ & $6.7(1)$ & 0.06 & 1.20 & in & 0.4 & 24 & 2.7 & 4 \\
\hline$\mu$ Ara d & 4.2 & 15.3 & G3IV-V & $5.2 \mathrm{E}-01$ & $5.1(1)$ & 0.06 & 0.92 & in & 2.8 & 4 & 2.7 & 4 \\
\hline Average & 3.5 & 13.2 & & $1.8 \mathrm{E}+00$ & 5.7 & 0.08 & 1.01 & & 1.7 & 6 & 1.6 & 6 \\
\hline Summary & \multicolumn{2}{|c|}{ Total: 14} & \multicolumn{2}{|c|}{$\mathrm{F}: \mathrm{G}: \mathrm{K}: \mathrm{M}=1: 2: 7: 3$} & & & & \multicolumn{2}{|c|}{ in:out $=11: 2$} & & & \\
\hline
\end{tabular}

Notes. ${ }^{(\S)}$ Planets that are also observable with $g=1.0 .^{(\dagger)}$ The radii of all the listed planets are predicted from the mass. The figures in the parentheses represent the methods to estimate $R_{\mathrm{p}}$ : (1) adopts the mass-radius relation for $M_{\mathrm{p}}>150 M_{\text {Earth }}\left(0.47 M_{\text {Jup }}\right) ;(2) 10 M_{\text {Earth }}\left(0.031 M_{\text {Jup }}\right)<$ $M_{\mathrm{p}} \leq 150 M_{\text {Earth }}$; and (3) $4 M_{\text {Earth }}\left(0.013 M_{\text {Jup }}\right)<M_{\mathrm{p}} \leq 10 M_{\text {Earth }}$. See text for details. ${ }^{(\ddagger)}$ This column describes whether the planet is located inside or outside the snow line. The snow line distance is estimated by Eq. (17) in Ida \& Lin (2005).

$\Delta t$ to $50 \mathrm{~h}$ (with $g=0.05$ ), the number of observable planets increases to 20 (Fig. 5).

Note that we have extracted observable planets from the already known planets. Further, observable planets are not necessarily limited to those in Table 2 , given that transit and radial velocity detection of exoplanets is biased toward edge-on systems, whereas direct observations can also find those in face-on systems.

\subsection{Notes on some of the observable planets}

Among the listed observable planets, Aldebaran $\mathrm{b}$ orbits the brightest $\operatorname{star}\left(m^{*}(J)=-2.1 \mathrm{mag}\right)$. The existence of a planetary companion with a mass of $6.47 \pm 0.53 M_{\text {Jup }}$ is considered to be the most plausible explanation for the observed radial velocity (RV) variations with a 629-day period (Hatzes et al. 2015). Owing to the extreme brightness of the host star, $\sigma_{P}^{\text {phot }}$ for the planet will be suppressed to a very low level $(\sim 0.2 \%)$. Aldebaran $b$, if it truly exists, may be one of the first targets for the spectro-polarimetry.

Gliese 876 (=GJ 876) is currently known to host four planets. Discoveries of planets "b" and "e" were made based on RV observations by Delfosse et al. (1998) and Marcy et al. (1998); and Rivera et al. (2010); respectively. It is expected that both of the planets can be observable even with $g=1.0$. The contrast plot for Gliese $876 \mathrm{~b}$ is shown in Figs. $6 \mathrm{c}$ and $\mathrm{d}$ for $g=1.0$ and 0.05 , respectively. The orbital distance of the snow line in the system is evaluated to be $0.30 \mathrm{AU}$ based on the mass of the host star $\left(0.334 M_{\odot}\right)$. Fortunately, the orbital distance of planet " $\mathrm{b}$ " $\left(a_{\mathrm{p}}=0.21 \mathrm{AU}\right)$ is estimated to be within the snow line, whereas that of planet "e" $(0.33 \mathrm{AU})$ is predicted to be beyond the line. Therefore, this system may be an optimal target to investigate the existence of $\mathrm{H}_{2} \mathrm{O}$ vapor on planets inside and outside the snow line.

\section{Discussions}

\subsection{Unconsidered factors}

In this paper, we explore the feasibility of spectro-polarimetric detection of $\mathrm{H}_{2} \mathrm{O}$ vapor on exoplanets by evaluating the $P$ errors, which arise mainly from the efficiency differences between polarization states, speckle noises, and photon noise. In this section, some unconsidered factors are discussed.

Influences from the circumstellar disks were not discussed. Min et al. (2012) pointed out that the central resolution element on a disk image can be polarized because of scattering from the innermost regions of the disk. This makes the central star appear to have an intrinsic polarization. However, the polarization degree was calculated to be less than $0.1 \%$ for a spatial element obtained through 30-40 m telescopes (Min et al. 2012). As we have assumed much greater interstellar and instrumental polarization (several percent, Table 1), consideration of this effect will not change the result of our feasibility estimates.

As another influence from the disks, we consider exo-zodical light around a planet image. For low-spatial-resolution images, exo-zodical light may obscure a planet image. Simulations by Cash et al. (2008) showed that intensity of an Earth twin in a solar system twin at $10 \mathrm{pc}$ was comparable to that of the surrounding exo-zodical light when observed through a $4.0 \mathrm{~m}$ diffraction-limited telescope (see their Fig. 1). However, as spatial resolution improves, a planetary image sharpens. When the same system is viewed through a $30-40 \mathrm{~m}$ telescope, intensity of the exo-zodical light per a spatial resolution element is approximately 50-100 times fainter than that of the Earth twin. Hence, we expect that this effect will not interfere with detection of a spectro-polarimetric feature with $\Delta P=10 \%$.

Keller (1996) mentioned the interference fringes due to multiple reflections between the retarder surfaces. Fringes on intensity spectra were observed at a level of $1 \times 10^{-5}$, which may lead 
(a)

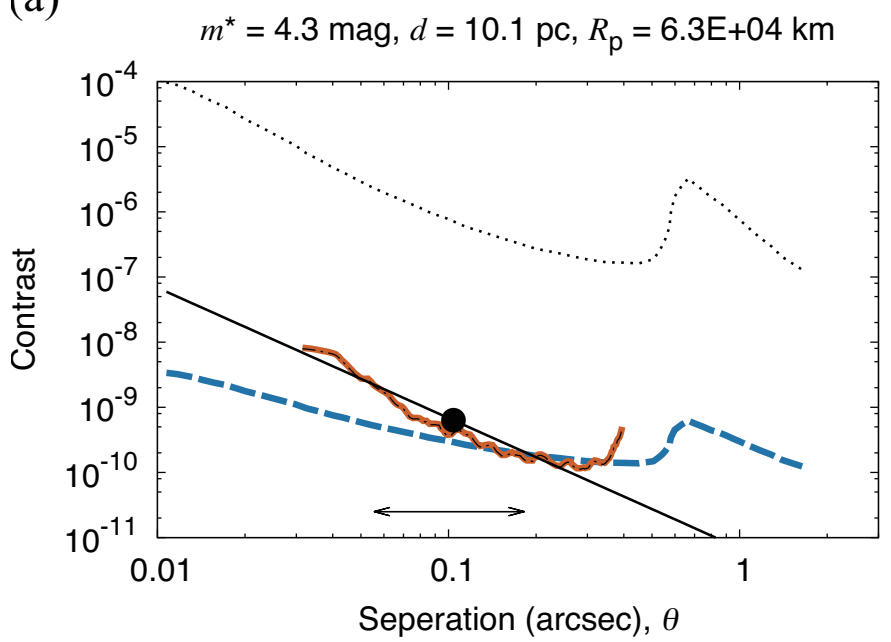

(c)

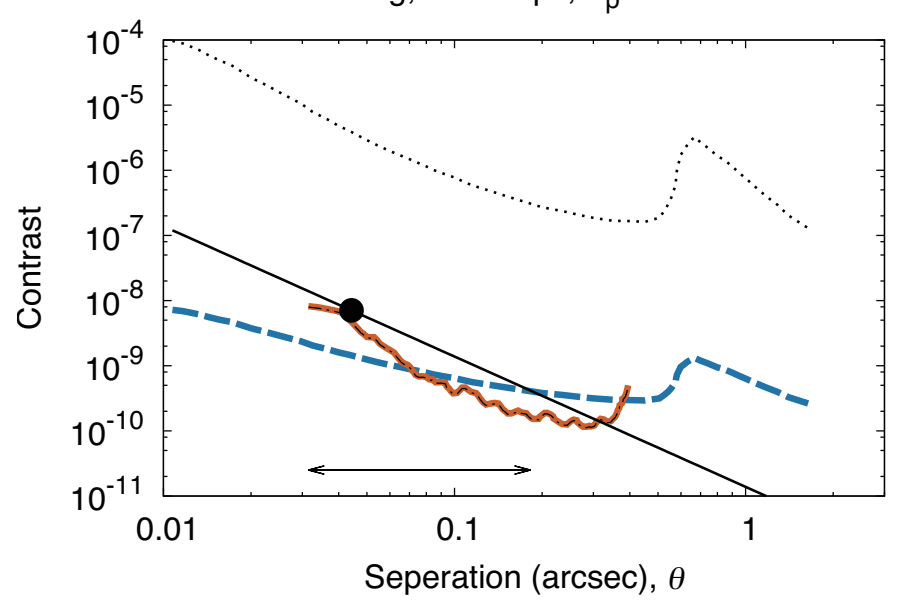

(b)

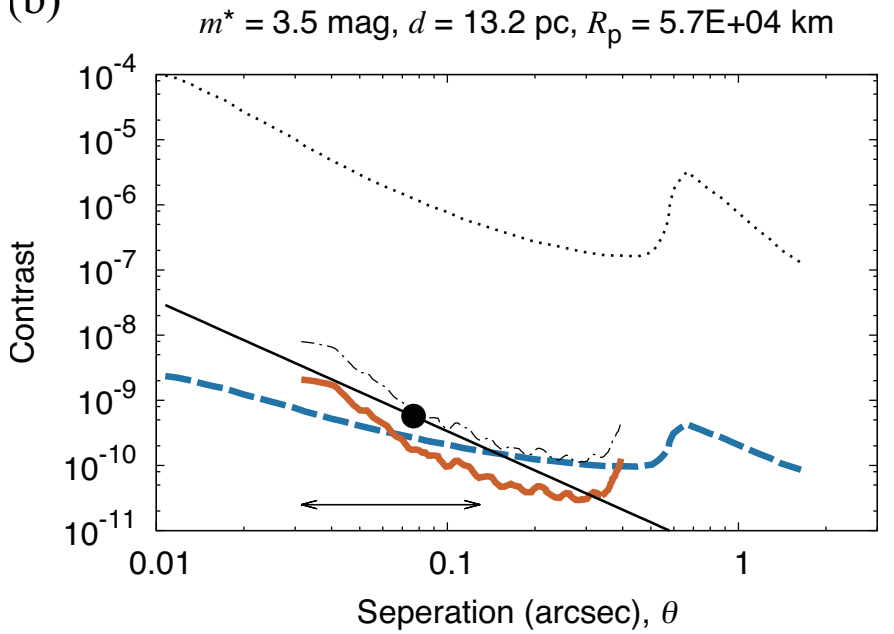

(d)

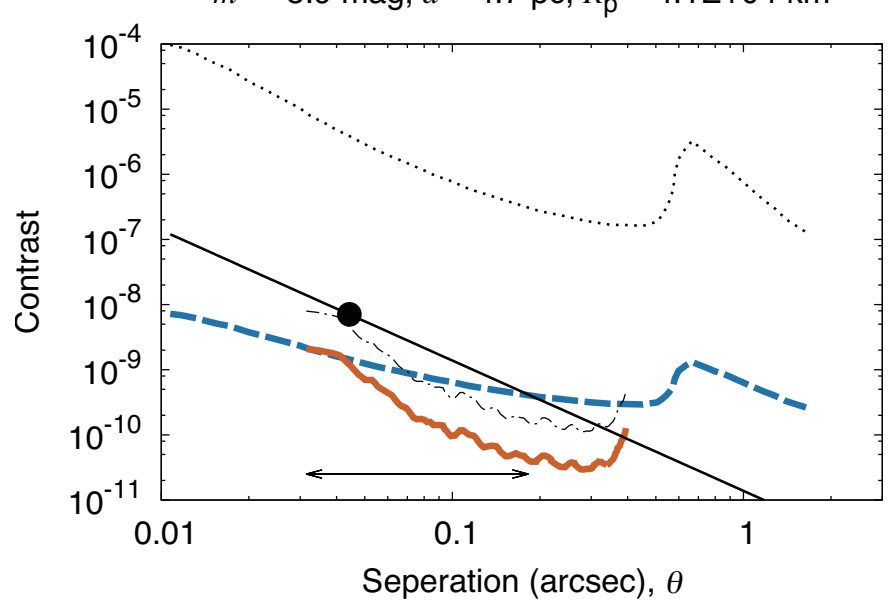

Fig. 6. Comparison of contrasts. Contrast performance limited by the speckle noises (bold solid lines) and by the photon noise (dashed lines) is compared with $\triangle P C_{\mathrm{p} / \mathrm{s}}$ (filled circles), that is, contrast of the polarized feature signal. a) a "typical" planet with averaged properties of the observable planets with $g=1.0$. b) same as a) except for $g=0.05$. c) Gliese $876 \mathrm{~b}$ with $g=1.0$. d) Gliese $876 \mathrm{~b}$ with $g=0.05$. The thin solid line in each panel is $\Delta P C_{\mathrm{p} / \mathrm{s}}(\theta)$ for a planet with the same radius. The polarization feature is detectable in the ranges of $\theta$ designated by the arrows. 3-sigma $C_{\text {det }}$ (dashed-dotted lines) and $C_{\text {raw }}$ (dotted lines) are also plotted for the reference.

to fringes on $P$ spectra at a few times $10^{-5}$. There are instrumental approaches and a data reduction method called the autoregressive model to reduce the fringes (Keller 1996). Although these approaches may not be perfect, we expect that the fringes will not pose a serious problem as long as the EPICS-IFS-like $C_{\text {det }}$ is achieved as we assume, because the degree of the fringe errors is expected to be much smaller than $\Delta P(=10 \%)$.

\subsection{Future works}

Sophisticated data reduction techniques may suppress the systematic bias more efficiently. We have adopted Eq. (1) to derive $P$, which is the simplest way. For a dual beam polarimeter, however, there are two other approaches: the "difference" and "ratio" methods (Keller 1996; Bagnulo et al. 2009). These methods can be applied by obtaining two sets of ordinary and extraordinary images while switching the polarization states (rotating the position angle of polarization by $90^{\circ}$ ) with a half-wave plate or a ferroelectric liquid crystal. It is anticipated that better $g$ will be achieved using these techniques. Evaluation of their effects is desirable but beyond the scope of this work
Obviously, laboratory and on-sky experiments are required to confirm and refine our estimates. In particular, determination and examination of $g$ is important. In this paper, we have parameterized $g$ as the combination of many uncertain factors including difference between transmittances for orthogonally polarized states, polarization aberrations, and wavefront aberrations. Knowledge of the dependence of $g$ on these factors will lead to minimization of $g$ and enhancement of detection contrast through a combination of the SSDI and PDI techniques.

Despite the remaining future works, this study has provided a basic framework to evaluate the feasibility of spectropolarimetric detection of atmospheric components and presented the instrumental requirements with regards to $C_{\text {raw }}, C_{\text {det }}, g$, and $\bar{k}$.

\section{Summary and concluding remarks}

Motivated by the scientific and technical benefits of spectropolarimetry for the reflected light from exoplanets, the feasibility of the spectro-polarimetric search for $\mathrm{H}_{2} \mathrm{O}$ vapor has been investigated. We formulated three types of errors in the measurement of the polarization degree: (a) $b_{P}^{\text {eff }}$, errors from different 
efficiencies (throughputs from the light source to the detector) between ordinary and extraordinary light paths; (b) $\sigma_{P}^{\text {spec }}$, errors caused by speckle noises; and (c) $\sigma_{P}^{\text {phot }}$, errors caused by photon noise from the scattered starlight. Based on the derived equations, we have calculated the requirements for instrumental contrasts to detect the enhanced feature of $\mathrm{H}_{2} \mathrm{O}$ at $\lambda_{\mathrm{abs}}=1.12 \mu \mathrm{m}$ with $P\left(\lambda_{\mathrm{cnt}}\right)=10 \%$ and $\Delta P=10 \%$. Furthermore, the number of observable planets was estimated, assuming availability of the E-ELT and a spectro-polarimeter with an EPICS-IFS-like contrast. The results show that 5 to 14 known planets are observable. Therefore, we remark that spectro-polarimetric characterization of exoplanetary atmospheres is feasible in principle at the coming age of ELTs. Since we have conducted simple analytical formulations, the framework we have developed will be easily adopted for feasibility evaluations with other settings, such as those using an instrument with a different contrast or a search for other molecular species.

Acknowledgements. We are deeply grateful to N. Murakami for discussion on the physical interpretation of instrumental contrasts. We also thank H. Kawahara for suggestions about the estimates of observable planets. We express our gratitude to the anonymous referee for helpful comments and suggestions. This work was supported by JSPS KAKENHI Grant Numbers 15K21296 and 25247021

\section{References}

Bagnulo, S., Landolfi, M., Landstreet, J. D., et al. 2009, PASP, 121, 993 Bailey, J., Ulanowski, Z., Lucas, P. W., et al. 2008, MNRAS, 386, 1016 Barman, T. 2007, ApJ, 661, L191

Barman, T. S., Macintosh, B., Konopacky, Q. M., \& Marois, C. 2011, ApJ, 733 65

Breckinridge, J. B., \& Oppenheimer, B. R. 2004, ApJ, 600, 1091

Breckinridge, J. B., Lam, W. S. T., \& Chipman, R. A. 2015, PASP, 127, 445

Cash, W., Oakley, P., Turnbull, M., et al. 2008, in Space Telescopes and Instrumentation 2008: Optical, Infrared, and Millimeter, Proc. SPIE, 7010 70101

de Juan Ovelar, M., Diamantopoulou, S., Roelfsema, R., et al. 2012, in Modeling, Systems Engineering, and Project Management for Astronomy V, Proc. SPIE 8449,844912

Delfosse, X., Forveille, T., Perrier, C., \& Mayor, M. 1998, A\&A, 331, 581

Dollfus, A. 1957, Suppléments aux Annales d'Astrophysique, 4, 3

Fossati, L., Bagnulo, S., Mason, E., \& Landi Degl'Innocenti, E. 2007, in The Future of Photometric, Spectrophotometric and Polarimetric Standardization, ed. C. Sterken, ASP Conf. Ser., 364, 503
Garufi, A., Quanz, S. P., Avenhaus, H., et al. 2013, A\&A, 560, A105 Hashimoto, J., Tamura, M., Muto, T., et al. 2011, ApJ, 729, L17 Hatzes, A. P., Cochran, W. D., Endl, M., et al. 2015, A\&A, 580, A31 Ida, S., \& Lin, D. N. C. 2005, ApJ, 626, 1045

Kasper, M., Beuzit, J.-L., Verinaud, C., et al. 2010, in SPIE Conf. Ser., 7735, 2 Keller, C. U. 1996, Sol. Phys., 164, 243

Keller, C. U., Schmid, H. M., Venema, L. B., et al. 2010, in SPIE Conf. Ser., 7735,6

Konopacky, Q. M., Barman, T. S., Macintosh, B. A., \& Marois, C. 2013, Science 339, 1398

Lenzen, R., Hartung, M., Brandner, W., et al. 2003, in Instrument Design and Performance for Optical/Infrared Ground-based Telescopes, eds. M. Iye, \& A. F. M. Moorwood, SPIE Conf. Ser., 4841, 944

Lord, S. D. 1992, A new software tool for computing Earth's atmospheric transmission of near- and far-infrared radiation, Tech. rep. (NASA)

Marcy, G. W., Butler, R. P., Vogt, S. S., Fischer, D., \& Lissauer, J. J. 1998, ApJ 505, L147

Marois, C., Doyon, R., Racine, R., \& Nadeau, D. 2000, PASP, 112, 91

McCullough, P. R., Crouzet, N., Deming, D., \& Madhusudhan, N. 2014, ApJ, 791, 55

Miles-Páez, P. A., Pallé, E. \& Zapatero Osorio, M. R. 2014, A\&A, 562, L5

Min, M., Canovas, H., Mulders, G. D., \& Keller, C. U. 2012, A\&A, 537, A75

Montañés-Rodriguez, P., Pallé, E., Goode, P. R., Hickey, J., \& Koonin, S. E. 2005, ApJ, 629, 1175

Rivera, E. J., Laughlin, G., Butler, R. P., et al. 2010, ApJ, 719, 890

Rodenhuis, M., Sprenger, B., \& Keller, C. U. 2012, in Ground-based and Airborne Instrumentation for Astronomy IV, Proc. SPIE, 8446, 84469

Rousset, G., Lacombe, F., Puget, P., et al. 2003, in Adaptive Optical System Technologies II, eds. P. L. Wizinowich, \& D. Bonaccini, SPIE Conf. Ser., 4839,140

Schneider, J., Dedieu, C., Le Sidaner, P., Savalle, R., \& Zolotukhin, I. 2011, A\&A, 532, A79

Sparks, W. B., \& Ford, H. C. 2002, ApJ, 578, 543

Stam, D. M. 2008, A\&A, 482, 989

Stam, D. M., Hovenier, J. W., \& Waters, L. B. F. M. 2004, A\&A, 428, 663

Sterzik, M. F., Bagnulo, S., \& Palle, E. 2012, Nature, 483, 64

Tamura, M., Hodapp, K., Takami, H., et al. 2006, in SPIE Conf. Ser., 6269

Tanii, R., Itoh, Y., Kudo, T., et al. 2012, PASJ, 64, 124

Thalmann, C., Schmid, H. M., Boccaletti, A., et al. 2008, in SPIE Conf. Ser., 7014, 3

Thalmann, C., Mulders, G. D., Janson, M., et al. 2015, ApJ, 808, L41

Tinetti, G., Vidal-Madjar, A., Liang, M.-C., et al. 2007, Nature, 448, 169

Traub, W. A., \& Oppenheimer, B. R. 2010, Direct Imaging of Exoplanets (Tucson AZ: University of Arizona Press), ed. S. Seager, 111

Trauger, J. T., \& Traub, W. A. 2007, Nature, 446, 771

Turnbull, M. C., Traub, W. A., Jucks, K. W., et al. 2006, ApJ, 644, 55

Weiss, L. M., \& Marcy, G. W. 2014, ApJ, 783, L6

Weiss, L. M., Marcy, G. W., Rowe, J. F., et al. 2013, ApJ, 768, 14

Wenger, M., Ochsenbein, F., Egret, D., et al. 2000, A\&AS, 143, 9

Whittet, D. C. B., Martin, P. G., Hough, J. H., et al. 1992, ApJ, 386, 562 
Appendix A: Derivation of Eqs. (4) and (5)

By substituting $I_{\chi}^{\mathrm{p}}$ in Eq. (2) into $I_{\chi}^{\mathrm{p}}$ in Eq. (1), the polarization degree with errors is written as

$$
\begin{aligned}
P^{\prime} & =\frac{I_{0}^{\prime \mathrm{p}}-I_{90}^{\prime}}{I_{0}^{\prime}{ }_{0}^{\mathrm{p}}+I_{90}^{\prime}} \\
& =\frac{\left(r_{0} I_{0}^{\mathrm{p}}+\epsilon_{0}\right)-\left(r_{90} I_{90}^{\mathrm{p}}+\epsilon_{90}\right)}{\left(r_{0} I_{0}^{\mathrm{p}}+\epsilon_{0}\right)+\left(r_{90} I_{90}^{\mathrm{p}}+\epsilon_{90}\right)} \\
& =\frac{\left(r_{0} I_{0}^{\mathrm{p}}-r_{90} I_{90}^{\mathrm{p}}\right)+\left(\epsilon_{0}-\epsilon_{90}\right)}{\left(r_{0} I_{0}^{\mathrm{p}}+r_{90} I_{90}^{\mathrm{p}}\right)+\left(\epsilon_{0}+\epsilon_{90}\right)} \\
& =\frac{\left[r I^{\mathrm{p}}\right]_{-}+\epsilon_{-}}{\left[r I^{\mathrm{p}}\right]_{+}}+\epsilon_{+} \\
& =\left(\frac{\left[r I^{\mathrm{p}}\right]_{-}}{\left[r I^{\mathrm{p}}\right]_{+}}+\frac{\epsilon_{-}}{\left[r I^{\mathrm{p}}\right]_{+}}\right)\left(1+\frac{\epsilon_{+}}{\left[r I^{\mathrm{p}}\right]_{+}}\right) \\
& \cong\left(\frac{\left[r I^{\mathrm{p}}\right]_{-}}{\left[r I^{\mathrm{p}}\right]_{+}}+\frac{\epsilon_{-}}{\left[r I^{\mathrm{p}}\right]_{+}}\right)\left(1-\frac{\epsilon_{+}}{\left[r I^{\mathrm{p}}\right]_{+}}\right) \\
& =\frac{\left[r I^{\mathrm{p}}\right]_{-}}{\left[r I^{\mathrm{p}}\right]_{+}}+\frac{\epsilon_{-}}{\left[r I^{\mathrm{p}}\right]_{+}}-\frac{\left[r I^{\mathrm{p}}\right]_{-}}{\left[r I^{\mathrm{p}}\right]_{+}} \frac{\epsilon_{+}}{\left[r I^{\mathrm{p}}\right]_{+}}-\frac{\epsilon_{-} \epsilon_{+}}{\left(\left[r I^{\mathrm{p}}\right]_{+}\right)^{2}} \\
& \cong \frac{\left[r I^{\mathrm{p}}\right]_{-}}{\left[r I^{\mathrm{p}}\right]_{+}}+\frac{\epsilon_{-}}{\left[r I^{\mathrm{p}}\right]_{+}}-\frac{\left[r I^{\mathrm{p}}\right]_{-}}{\left[r I^{\mathrm{p}}\right]_{+}} \frac{\epsilon_{+}}{\left[r I^{\mathrm{p}}\right]_{+}},
\end{aligned}
$$

where $\left|\epsilon_{\chi}\right| \ll r_{\chi} I_{\chi}^{\mathrm{p}}$ is assumed in the approximations and the following replacements are used:

$$
\begin{aligned}
{\left[r I^{\mathrm{p}}\right]_{+} } & \equiv r_{0} I_{0}^{\mathrm{p}}+r_{90} I_{90}^{\mathrm{p}} \cong 2 \bar{r} \overline{\mathrm{p}} \\
{\left[r I^{\mathrm{p}}\right]_{-} } & \equiv r_{0} I_{0}^{\mathrm{p}}-r_{90} I_{90}^{\mathrm{p}}, \\
\epsilon_{+} & \equiv \epsilon_{0}+\epsilon_{90}, \\
\epsilon_{-} & \equiv \epsilon_{0}-\epsilon_{90} .
\end{aligned}
$$

We introduce the following parameters:

$$
\begin{aligned}
\rho & \equiv r_{90} / r_{0}-1, \\
l & \equiv I_{90}^{\mathrm{p}} / I_{0}^{\mathrm{p}} .
\end{aligned}
$$

With them, we have

$$
\begin{aligned}
\frac{\left[r I^{\mathrm{p}}\right]_{-}}{\left[r I^{\mathrm{P}}\right]_{+}} & =\frac{1-\frac{r_{90} I_{90}^{\mathrm{p}}}{r_{0} \mathrm{I}_{0}^{\mathrm{p}}}}{1+\frac{r_{90} I_{90}^{\mathrm{p}}}{r_{0} I_{0}^{\mathrm{p}}}} \\
& =\frac{1-(1+\rho) l}{1+(1+\rho) l} \\
& =(1-l-\rho l)(1+l+\rho l)^{-1} \\
& =(1-l-\rho l)(1+l)^{-1}\left(1+\frac{\rho l}{1+l}\right)^{-1} \\
& \cong(1-l-\rho l)(1+l)^{-1}\left(1-\frac{\rho l}{1+l}\right) \\
& =(1+l)^{-2}(1-l-\rho l)(1+l-\rho l) \\
& =(1+l)^{-2}\left[(1-l)(1+l)-2 \rho l+\rho^{2} l^{2}\right] \\
& \cong(1+l)^{-2}[(1-l)(1+l)-2 \rho l] \\
& =\frac{1-l}{1+l}-\frac{2 \rho l}{(1+l)^{2}} \\
& =P-\frac{\rho\left(1-P^{2}\right)}{2} \\
& =P-\frac{1}{2}\left(\frac{r_{90}}{r_{0}}-1\right)\left(1-P^{2}\right),
\end{aligned}
$$

where $\rho \ll 1$ is assumed in the approximations. In the last transformation,

$$
\begin{aligned}
P & =\frac{1-l}{1+l}, \\
1-P^{2} & =\frac{4 l}{(1+l)^{2}},
\end{aligned}
$$

are used. By substituting Eq. (A.8) into Eq. (A.1), we obtain

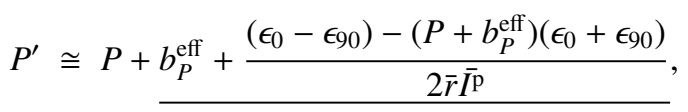

where

$b_{P}^{\mathrm{eff}} \equiv-\frac{1}{2}\left(\frac{r_{90}}{r_{0}}-1\right)\left(1-P^{2}\right)$.

The underlined part is the error in $P$, that is, $\mathcal{E}_{p}$. Since $r_{\chi}=$ $\mathcal{S}_{\chi} \mathcal{T}_{\chi} k_{\chi}$, we have

$$
\begin{aligned}
\frac{r_{90}}{r_{0}} & =\frac{\mathcal{S}_{90}}{\mathcal{S}_{0}} \frac{\mathcal{T}_{90}}{\mathcal{T}_{0}} \frac{k_{90}}{k_{0}} \\
& \cong\left(1-\frac{2 \delta \mathcal{S}}{\overline{\mathcal{S}}}\right)\left(1-\frac{2 \delta \mathcal{T}}{\overline{\mathcal{T}}}\right)\left(1-\frac{2 \delta k}{\bar{k}}\right) \\
& \cong 1-2\left(\frac{\delta \mathcal{S}}{\overline{\mathcal{S}}}+\frac{\delta \mathcal{T}}{\overline{\mathcal{T}}}+\frac{\delta k}{\bar{k}}\right) .
\end{aligned}
$$

Thus, we can also write $b_{P}^{\mathrm{eff}}$ as

$$
b_{P}^{\mathrm{eff}}=\left(\frac{\delta \mathcal{S}}{\overline{\mathcal{S}}}+\frac{\delta \mathcal{T}}{\overline{\mathcal{T}}}+\frac{\delta k}{\bar{k}}\right)\left(1-P^{2}\right)
$$

\section{Appendix B: Width of the spectral speckle features}

The FWHM of a spectral speckle feature, which overlays the real feature on $I^{\prime \mathrm{p}}$ and $P$ spectra at $\lambda_{\mathrm{abs}}$ is

$\Delta \lambda^{\mathrm{spec}}=\frac{\Delta \theta}{\theta_{\mathrm{p}}} \lambda_{\mathrm{abs}}$

where $\Delta \theta$ is the FWHM of a point source image and $\theta_{\mathrm{p}}$ is the separation of the planet from the star. Equation (B.1) shows that the width of the spectral speckle features becomes narrower as the planet is more distant from the star. In the case of the E-ELT (aperture diameter $D=39 \mathrm{~m}$ ), we have $\Delta \theta \cong \lambda_{\text {abs }} / D \cong 0.006^{\prime \prime}$. When $\theta_{\mathrm{p}}$ is taken as the inner working angle (IWA) of the EPICSIFS, $0.03^{\prime \prime}$ (Kasper et al. 2010) for example, we have $\Delta \lambda^{\text {spec }} \cong$ $0.22 \mu \mathrm{m}$, which is wide enough to be distinguished from the real feature with a FWHM $(\Delta \lambda)$ of $\sim 0.05 \mu \mathrm{m}$ (Miles-Páez et al. 2014). However, when $\theta_{\mathrm{p}}$ is $0.1^{\prime \prime}, \Delta \lambda^{\mathrm{spec}}$ is $\sim 0.07 \mu \mathrm{m}$, which is comparable with $\Delta \lambda$. As long as the spectro-polarimeter has a minimum wavelength resolution to detect the real feature, we should perceive that, except for a planet near the IWA, the spurious spectral features by the speckles cannot be distinguished from the real feature based on the feature widths. Therefore, it must be guaranteed that the strength of the speckle features on the $P$ spectrum is small enough compared to that of the real feature $(\Delta P)$.

\section{Appendix C: Raw contrast and detection contrast}

In this appendix, the raw contrast $\left(C_{\text {raw }}\right)$ and the detection contrast $\left(C_{\text {det }}\right)$ of a high-contrast instrument are explained and formulated using wavefront ripples of the electric field. 
The intensity (equivalent to the number of photons per unit area per unit time) of speckle noises for an (extra)ordinary component varies with position (separation from the star $\theta$ and position angle $\psi$ ) and time $t$. Speckle noises arise from ripples of the electromagnetic wavefront. Extreme AO corrects the ripples to the extent $\phi \sim \frac{2 \pi}{10}$ (or $\frac{\lambda}{10}$ in a length scale) or more, but cannot perfectly remove them. $\phi$ can be separated into random $\left(\phi_{\text {rand }}\right)$ and quasi-static $\left(\phi_{\mathrm{qst}}\right)$ components. $\phi_{\text {rand }}$ is mainly caused by atmospheric turbulence and varies randomly on short time-scales of a few to ten milliseconds. $\phi_{\mathrm{qst}}$ comes from optical imperfections of the instrument and evolves on time-scales of minutes to hours. $\phi_{\mathrm{qst}}$ can be divided into two components: one from a telescope to a coronagraph (including the coronagraph; $\left.\phi_{\mathrm{qst} 1}\right)$ and the other after the coronagraph $\left(\phi_{\mathrm{qst} 2}\right.$; see Fig. 1$)$.

We examine the stellar electric field immediately after the coronagraph, $E_{\mathrm{c}}$. Here, we take a shearing interferometric nuller as a coronagraph for a simple analytical discussion, but the discussion below can be applied to other types of coronagraphs. In the nuller, incident light is separated into two rays $E_{1}=A \mathrm{e}^{\mathrm{i} \phi_{1}}$ and $E_{2}=A \mathrm{e}^{\mathrm{i}\left(\phi_{2}+\pi\right)}$, where $A$ is a constant, and $\phi_{1}$ and $\phi_{2}$ are the phase perturbations, which are assumed to be small $\left(\left|\phi_{1}\right|,\left|\phi_{2}\right| \ll 1\right) . E_{\mathrm{c}}$ can be written as

$$
\begin{aligned}
E_{\mathrm{c}} & =E_{1}+E_{2}=A \mathrm{e}^{\mathrm{i} \phi_{1}}-A \mathrm{e}^{\mathrm{i} \phi_{2}} \\
& =A \mathrm{e}^{\mathrm{i} \phi_{1}}\left(1-\mathrm{e}^{\mathrm{i}\left(\phi_{2}-\phi_{1}\right)}\right)=A \mathrm{e}^{\mathrm{i} \phi_{1}}\left(1-\mathrm{e}^{\mathrm{i} \Delta \phi}\right) \\
& \cong A\left(1+\mathrm{i} \phi_{1}\right)(-\mathrm{i} \Delta \phi)=A\left(-i \Delta \phi+\phi_{1} \Delta \phi\right)=A \phi_{\mathrm{c}}
\end{aligned}
$$

where $\Delta \phi \equiv \phi_{2}-\phi_{1}$. As for $\phi_{\mathrm{c}}$, we find

$\phi_{\mathrm{c}} \equiv-\mathrm{i} \Delta \phi+\phi_{1} \Delta \phi \cong-\mathrm{i} \Delta \phi=-\mathrm{i}\left(\Delta \phi_{\text {rand }}+\Delta \phi_{\mathrm{qst} 1}\right)$,

where we split $\Delta \phi$ into random $\left(\Delta \phi_{\text {rand }}\right)$ and quasi-static $\left(\Delta \phi_{\text {ast1 } 1}\right)$ components. When the static perturbation $\phi_{\mathrm{qst} 2}$ is added to $E_{\mathrm{c}}$, it becomes

$$
\begin{aligned}
E_{\mathrm{c}}^{\prime} & =E_{\mathrm{c}} \mathrm{e}^{\mathrm{i} \phi_{\mathrm{qs} 2}} \cong A \phi_{\mathrm{c}}\left(1+\mathrm{i} \phi_{\mathrm{qst} 2}\right)=A\left(\phi_{\mathrm{c}}+\mathrm{i} \phi_{\mathrm{c}} \phi_{\mathrm{qst} 2}\right) \\
& \cong A \phi_{\mathrm{c}}=-\mathrm{i} A\left(\Delta \phi_{\text {rand }}+\Delta \phi_{\mathrm{qs} 1}\right) .
\end{aligned}
$$

Thus, $\phi_{\mathrm{qst} 2}$ is negligible. The electric field at the following image plane is

$$
\begin{aligned}
E_{\mathrm{d}} & =\mathrm{FT}\left(E_{\mathrm{c}}^{\prime}\right)=\mathrm{iFT}(A) * \mathrm{FT}\left(\Delta \phi_{\text {rand }}+\Delta \phi_{\mathrm{qst} 1}\right) \\
& =\mathrm{iFT}(A) *\left[\mathrm{FT}\left(\Delta \phi_{\text {rand }}\right)+\mathrm{FT}\left(\Delta \phi_{\mathrm{qst} 1}\right)\right],
\end{aligned}
$$

where FT() represents the Fourier transform.

For an incident field $A \mathrm{e}^{\mathrm{i} \phi}$, the ratio of speckle intensity to stellar intensity, or contrast $C$, is given by

$C=\left(\frac{\pi h}{\lambda}\right)^{2}$, where the peaks and valleys of the wavefront ripple have values (length) $\pm h$ (Traub \& Oppenheimer 2010, Eq. (121)). The phase perturbation is described as

$\phi(x)=a^{\prime} \cos \left(2 \pi x / x_{0}+\alpha^{\prime}\right)+\mathrm{i} b^{\prime} \cos \left(2 \pi x / x_{0}+\beta^{\prime}\right)$,

where $x$ is a position at the pupil plane and $x_{0}$ is a typical spatial period of the ripples. $a^{\prime}, \alpha^{\prime}, b^{\prime}$, and $\beta^{\prime}$ are constants with units of radians. $a^{\prime}$ is related to $h$ by

$a^{\prime}=2 \pi h / \lambda$.

See Eqs. (108), (109) and the related text in Traub \& Oppenheimer (2010) for more details.

In the case being studied, the raw contrast is

$C_{\text {raw }}=\left(\frac{\pi \sqrt{h_{\text {rand }}^{2}+h_{\mathrm{qst} 1}^{2}}}{\lambda}\right)^{2}=\left(\frac{\pi h_{\text {rand }}}{\lambda}\right)^{2}+\left(\frac{\pi h_{\mathrm{qst} 1}}{\lambda}\right)^{2}$,

where $h_{\text {rand }}$ and $h_{\mathrm{qst} 1}$ are $h$ for $\Delta \phi_{\text {rand }}$ and $\Delta \phi_{\mathrm{qst}}$, respectively. As $h_{\mathrm{qst} 1}$ can be suppressed greatly by a deformable mirror in the AO, it is safe to assume $h_{\text {rand }} \gg h_{\mathrm{qst1}}$ in their typical values. Thus, we find

$C_{\text {raw }} \cong\left(\frac{\pi h_{\text {rand }}}{\lambda}\right)^{2}$.

$C_{\text {raw }}$ is a ratio of instantaneous intensities or photon rates. When we integrate photons on a detector for a certain duration $\Delta t$, the effective $h_{\text {rand }}\left(h_{\text {rand,e }}\right)$ will be

$h_{\text {rand,e }}=h_{\text {rand }} \sqrt{\frac{t_{0}}{\Delta t}}$,

where $t_{0}$ is a typical time-scale of $\Delta \phi_{\text {rand }}$, which is a few to ten milliseconds. With a sufficient $\Delta t, h_{\text {rand,e }}$ is much smaller than $h_{\mathrm{qst} 1}$ (if $h_{\text {rand }} \sim 10^{-4} \lambda$ and $h_{\mathrm{qst} 1} \sim 10^{-6} \lambda$, then $h_{\text {rand,e }}<h_{\mathrm{qst} 1}$ for $\Delta t>100 \mathrm{~s})$. The detection contrast $C_{\text {det }}$ is defined as the contrast achieved with a sufficient $\Delta t$, which is

$C_{\mathrm{det}}=\left(\frac{\pi h_{\mathrm{qst} 1}}{\lambda}\right)^{2}$.

According to experiments by Trauger \& Traub (2007), it is possible to reach $C_{\mathrm{det}}=10^{-10}$ in space. In this paper, $C_{\mathrm{det}}$ is referred from the values for a ground-based instrument EPICS-IFS Kasper et al. (2010; see our Fig. 6). 